\title{
Unfold: An integrated toolbox for overlap correction, non- linear modeling, and regression-based EEG analysis
}

\author{
Benedikt V Ehinger ${ }^{\text {Corresp., 1, } 2}$, Olaf Dimigen ${ }^{\text {Corresp. } 3}$ \\ 1 Institute of Cognitive Science, Universität Osnabrück, Osnabrück, Germany \\ 2 Donders Institute for Brain, Cognition and Behaviour, Radboud University, Nijmegen, Netherlands \\ 3 Department of Psychology, Humboldt-Universität zu Berlin, Berlin, Germany \\ Corresponding Authors: Benedikt V Ehinger, Olaf Dimigen \\ Email address: behinger@uos.de, olaf.dimigen@hu-berlin.de
}

Electrophysiological research with event-related brain potentials (ERPs) is increasingly moving from simple, strictly orthogonal stimulation paradigms towards more complex, quasi-experimental designs and naturalistic situations that involve fast, multisensory stimulation and complex motor behavior. As a result, electrophysiological responses from subsequent events often overlap with each other. In addition, the recorded neural activity is typically modulated by numerous covariates, which influence the measured responses in a linear or nonlinear fashion. Examples of paradigms where systematic temporal overlap variations and low-level confounds between conditions cannot be avoided include combined EEG/eye-tracking experiments during natural vision, fast multisensory stimulation experiments, and mobile brain/body imaging studies. However, even "traditional", highly controlled ERP datasets often contain a hidden mix of overlapping activity (e.g. from stimulus onsets, involuntary microsaccades, or button presses) and it is helpful or even necessary to disentangle these components for a correct interpretation of the results. In this paper, we introduce unfold, a powerful, yet easy-to-use MATLAB toolbox for regression-based EEG analyses that combines existing concepts of massive univariate modeling ("regression ERPs"), linear deconvolution modeling, and non-linear modeling with the generalized additive model (GAM) into one coherent and flexible analysis framework. The toolbox is modular, compatible with EEGLAB and can handle even large datasets efficiently. It also includes advanced options for regularization and the use of temporal basis functions (e.g. Fourier sets). We illustrate the advantages of this approach for simulated data as well as data from a standard face recognition experiment. In addition to traditional and non-conventional EEG/ERP designs, unfold can also be applied to other overlapping physiological signals, such as pupillary or electrodermal responses. It is available as open-source software at http://www.unfoldtoolbox.org . 


\section{Unfold: An integrated toolbox for overlap correction, non- 2 linear modeling, and regression-based EEG analysis}

3 Benedikt V. Ehinger ${ }^{1,2 *}$ \& Olaf Dimigen ${ }^{3}$

$4 \quad{ }^{1}$ Institute of Cognitive Science, Universität Osnabrück, Osnabrück, Germany

$5 \quad{ }^{2}$ Donders Institute for Brain, Cognition and Behaviour, Radboud University, Nijmegen,

6 Netherlands

$7 \quad 3$ Department of Psychology, Humboldt-Universität zu Berlin, Berlin, Germany

9 Corresponding Author

10 Benedikt Ehinger (behinger@uos.de)

11 Wachsbleiche 27

1249089 Osnabrück

13 Tel. +49 541 969-2245

$14 \quad$ Fax +49 $541969-2596$

16 Co-Corresponding Author

17 Olaf Dimigen (olaf.dimigen@hu-berlin.de)

18 Unter den Linden 6

1910099 Berlin

20 Tel. +49 $302093-4849$

21 Fax +49 $302093-4910$

Author note: The authors would like to thank Bernhard Spitzer for helpful discussions about deconvolution models, as well as Peter König, Guang Ouyang, Ashima Keshava, and Scott Makeig for comments on earlier versions of this work. Data and code to reproduce Figure 10 can be found under https://osf.io/wbz7x. 
ABSTRACT

Electrophysiological research with event-related brain potentials (ERPs) is increasingly moving from simple, strictly orthogonal stimulation paradigms towards more complex, quasiexperimental designs and naturalistic situations that involve fast, multisensory stimulation and complex motor behavior. As a result, electrophysiological responses from subsequent events often overlap with each other. In addition, the recorded neural activity is typically modulated by numerous covariates, which influence the measured responses in a linear or nonlinear fashion. Examples of paradigms where systematic temporal overlap variations and low-level confounds between conditions cannot be avoided include combined EEG/eye-tracking experiments during natural vision, fast multisensory stimulation experiments, and mobile brain/body imaging studies. However, even "traditional", highly controlled ERP datasets often contain a hidden mix of overlapping activity (e.g. from stimulus onsets, involuntary microsaccades, or button presses) and it is helpful or even necessary to disentangle these components for a correct interpretation of the results. In this paper, we introduce unfold, a powerful, yet easy-to-use MATLAB toolbox for regression-based EEG analyses that combines existing concepts of massive univariate modeling ("regression ERPs"), linear deconvolution modeling, and non-linear modeling with the generalized additive model (GAM) into one coherent and flexible analysis framework. The toolbox is modular, compatible with EEGLAB and can handle even large datasets efficiently. It also includes advanced options for regularization and the use of temporal basis functions (e.g. Fourier sets). We illustrate the advantages of this approach for simulated data as well as data from a standard face recognition experiment. In addition to traditional and non-conventional EEG/ERP designs, unfold can also be applied to other overlapping physiological signals, such as pupillary or electrodermal responses. It is available as open-source software at http://www.unfoldtoolbox.org.

Keywords: EEG, ERP, overlap correction, linear modeling of EEG, regression-ERP (rERP), generalized additive model (GAM), non-linear modeling, regression splines, regularization 
55

56

57

58

59

60

61

62

63

64

65

66

67

68

69

\section{INTRODUCTION}

Event-related brain responses in the electroencephalogram (EEG) are traditionally studied in strongly simplified and strictly orthogonal stimulus-response paradigms. In many cases, each experimental trial involves only a single, tightly controlled stimulation and a single manual response. In recent years, however, there has been rising interest in recording brain-electric activity also in more complex paradigms and naturalistic situations. Examples include laboratory studies with fast and concurrent streams of visual, auditory, and tactile stimuli (e.g. Spitzer, Blankenburg, \& Summerfield, 2016), experiments that combine EEG recordings with eye-tracking recordings during natural vision (e.g. Dimigen, Sommer, Hohlfeld, Jacobs, \& Kliegl, 2011), EEG studies in virtual reality (e.g. Ehinger et al., 2014) or mobile brain/body imaging studies that investigate realworld interactions of freely moving participants (e.g. Gramann, Jung, Ferris, Lin, \& Makeig, 2014). There are two main problems in these types of situations: Overlapping neural responses from subsequent events and complex influences of nuisance variables that cannot be fully controlled. However, even traditional ERP experiments often contain a mixture of overlapping neural responses, for example from stimulus onsets, involuntary microsaccades, or manual button presses.

Appropriate analysis of such datasets requires a paradigm shift away from simple averaging techniques towards more sophisticated, regression-based approaches (Amsel, 2011; Pernet, Chauveau, Gaspar, \& Rousselet, 2011; N. J. Smith \& Kutas, 2015b; Frömer, Maier, \& Abdel Rahman, 2018; Hauk, Davis, Ford, Pulvermüller, \& Marslen-Wilson, 2006; Van Humbeeck, Meghanathan, Wagemans, van Leeuwen, \& Nikolaev, 2018) that can deconvolve overlapping potentials and also control or model the effects of both linear and non-linear covariates on the neural response. Importantly, the basic algorithms to deconvolve overlapping signals and to model the influences of both linear and non-linear covariates already exist. However, there is not yet a toolbox that integrates all of the necessary methods in one coherent workflow.

In the present paper we introduce unfold, an open source, easy-to-use, and flexible MATLAB toolbox written to facilitate the use of advanced deconvolution models and spline regression in ERP research. It performs these calculations efficiently even for large models and datasets and allows to run complex models with a few lines of codes. The toolbox is programmed in a modular fashion, meaning that intermediate analysis steps can be readily inspected and modified by the 
85 user if needed. It is also fully documented, can employ regularization, can model both linear and 86 nonlinear effects using spline regression, and is compatible with EEGLAB (Delorme \& Makeig,

87 2004) a widely used toolbox to preprocess electrophysiological data that offers importers for many 88 other biometric data formats, including eye-tracking and pupillometric data as well. unfold offers 89 built-in functions to visualize the model coefficients (betas) of each predictor as waveforms or scalp topographies (i.e. "regression-ERPs", rERPs, Burns, Bigdely-Shamlo, Smith, KreutzDelgado, \& Makeig, 2013; N. J. Smith \& Kutas, 2015a). Alternatively, results can be easily exported as plain text or transferred to other toolboxes like EEGLAB or Fieldtrip (Oostenveld, Fries, Maris, \& Schoffelen, 2011). For statistical analyses at the group level, that is second-level statistics, the resulting rERPs can be treated just like any other subject-level ERPs. As one suggestion, unfold integrates threshold-free cluster enhancement (TFCE) permutation tests for this purpose (S. M. Smith \& Nichols, 2009; Mensen \& Khatami, 2013)

In the following, we first briefly summarize some key concepts of regression-based EEG analysis, with an emphasis on linear deconvolution, spline regression, and temporal basis functions. We then describe the unfold toolbox that combines these concepts into one coherent framework. Finally, we illustrate its application to simulated data as well as real data from a standard ERP experiment. In particular, we will go through the typical steps to run and analyze a deconvolution model, using the data of a standard face recognition ERP experiment that contains overlapping potentials from three different sources: from stimulus onsets, from button presses, and from microsaccades, small eye movements that were involuntarily made by the participants during the task. We also give detailed descriptions of the features of the toolbox, including practical recommendations, simulation results, and advanced features such as regularization options or the use of temporal basis functions. We hope that our toolbox will both improve the understanding of traditional EEG datasets (e.g. by separating stimulus- and response-related components) as well as facilitate electrophysiological analyses in complex or (quasi-)natural situations, such as in combined eye-tracking/EEG and mobile brain/body imaging studies.

112 Before we introduce a real dataset, let us first consider a simulated simple EEG/ERP study

113 to illustrate the possibilities of the deconvolution approach. For this, let's imagine a typical 114 stimulus-discrimination task with two conditions (Figure 1): Participants are shown pictures of 
115 faces or houses and asked to classify the type of stimulus with a button press. Because this response

116 is speeded, motor activity related to the preparation and execution of the manual response will

117 overlap with the activity elicited by stimulus onset. Furthermore, we also assume that the mean

118 reaction time (RTs) differs between the conditions, as it is the case in most experiments. In our

119 example, if face pictures are on average classified faster than houses pictures (Figure 1C), then a

120 different overlap between stimulus- and response-related potentials will be observed in the two

121 conditions. Importantly, as Figure $1 \mathrm{~F}$ shows, this will result in spurious conditions effects due to

122 the varying temporal overlap alone, which can be easily mistaken for genuine differences in the

123 brain's processing of houses and faces.

124 Human faces are also complex, high-dimensional stimuli with numerous properties that are

125 difficult to perfectly control and orthogonalize in any given study. For simplicity, we assume here

126 that the average luminance of the stimuli was not perfectly matched between conditions and is

127 slightly, but systematically, higher for faces than houses (Figure 1D). From previous studies, we

128 know that the amplitude of the P1 visually-evoked potential increases as a non-linear $(\log )$ function

129 of the luminance of the presented stimulus (Halliday, 1982), and thus we also simulate a

130 logarithmic effect of luminance on the P1 of the stimulus-aligned ERP (Figure 1E), which creates

131 another spurious condition difference (Figure 1G) in addition to that of varying response times

132 (Figure 1F).

133 Panels $\mathrm{G}$ and $\mathrm{H}$ of Figure 1 show the same data modeled with unfold. Fortunately, with

134 deconvolution modeling, we can not only remove the overlap effect (Figure 1G), but by including

135 luminance as a non-linear predictor, we simultaneously also control the influence of this covariate

136 (Figure 1H). How this is done is explained in more detail in the following.

\section{Existing deconvolution approaches}

138 Deconvolution methods for EEG have existed for some time (Hansen, 1983; Eysholdt \& 139 Schreiner, 1982), but most older deconvolution approaches show severe limitations in their 140 applicability. They are either restricted to just two different events (Hansen, 1983; Zhang, 1998), 141 require special stimulus sequences (Eysholdt \& Schreiner, 1982; Marsh, 1992; Delgado \& 
142 Ozdamar, 2004; Jewett et al., 2004; Wang, Özdamar, Bohórquez, Shen, \& Cheour, 2006), rely on 143 semi-automatic, iterative methods like ADJAR (Woldorff, 1993) that can be slow or difficult to 144 converge (Talsma \& Woldorff, 2004; Kristensen, Rivet, \& Guérin-Dugué, 2017), or were tailored 145 for special applications. In particular, the specialized RIDE algorithm (Ouyang, Herzmann, Zhou, 146 \& Sommer, 2011; Ouyang, Sommer, \& Zhou, 2015) offers a unique feature in that it able to 147 deconvolve time-jittered ERP components even in the absence of a designated event marker. 148 However, while RIDE has been successfully used to separate stimulus- and response-related ERP 149 components (Ouyang et al., 2011, 2015); it does not support continuous predictors and is intended 150 for a small number of overlapping events.

151 In recent years, an alternative deconvolution method based on the linear model has been 152 proposed and successfully applied to the overlap problem (Lütkenhöner, 2010; S. Dandekar, 153 Privitera, Carney, \& Klein, 2012; Litvak, Jha, Flandin, \& Friston, 2013; Spitzer et al., 2016; 154 Kristensen, Guerin-Dugué, \& Rivet, 2017; Kristensen, Rivet, et al., 2017; Sassenhagen, 2018; 155 Cornelissen, Sassenhagen, \& Võ, 2019; Coco, Nuthmann, \& Dimigen, 2018; Bigdely-Shamlo et 156 al., 2018). This deconvolution approach was first applied extensively to fMRI data (Dale \& 157 Buckner, 1997) where the slowly varying BOLD signal overlaps between subsequent events. 158 However, in fMRI, the shape of the BOLD response is well-known and this prior knowledge 159 allows the researcher to use model-based deconvolution. If no assumptions about the response 160 shape (i.e. the kernel) are made, the approach used in fMRI is closely related to the basic linear 161 deconvolution approach discussed below.

162

163

164

165

166

167

168

169

170

171

\section{Deconvolution within the linear model}

With deconvolution techniques, overlapping EEG activity is understood as the linear convolution of experimental event latencies with isolated neural responses (Figure 1B). The inverse operation is deconvolution, which recovers the unknown isolated neural responses given only the measured (convolved) EEG and the latencies of the experimental events (Figure 1H). Deconvolution is possible if the subsequent events in an experiment occur with varying temporal overlap, in a varying temporal sequence, or both. In classical experiments, stimulus-onset asynchronies and stimulus sequences can be varied experimentally and the latencies of motor actions (such as saccades or button presses) also vary naturally. This varying overlap allows for modeling of the unknown isolated responses, assuming that the overlapping signals add up 
172 linearly. More specifically, we assume (1) that the electrical fields generated by the brain sum 173 linearly (a justified assumption, see Nunez \& Srinivasan, 2006) and (2) that the overlap, or interval

174 between events, does not influence the computations occurring in the brain - and therefore the 175 underlying waveforms (see also Discussion).

176 The benefits of this approach are numerous: The experimental design is not restricted to 177 special stimulus sequences, multiple regression allows modeling of an arbitrary number of 178 different events, and the mathematical properties of the linear model are well understood.

\section{Linear deconvolution}

180 The classic massive univariate linear model, without overlap correction, is applied to epoched EEG data and can be written as:

$$
\mu_{i, \tau}=X_{i} \beta_{\tau} \text { with } y_{i, \tau} \sim \operatorname{normal}\left(\mu_{i, \tau}, \sigma_{\tau}\right)
$$

Here, $X$ is the design matrix. It has i rows (each describing one instance of an event of type e) and c columns (each describing the status of one predictor).

Furthermore, let $\tau$ be the "local time" relative to onset of the event (e.g. -100 to +500 sampling points). $\mu_{i, \tau}$ is the expected (average) EEG signal measured after event $i$ that we wish to predict at a given time point $\tau$ relative to the event onset. $\beta$ is a vector of unknown parameters that we wish to estimate for each time point in the epoched EEG data. Importantly, therefore, this approach fits a separate linear model at each time point $\tau$.

A single entry will be referred by lowercase $x_{i, c}$.

In contrast, with linear deconvolution we enter the continuous EEG data into the model. We then make use of the knowledge that each observed sample of the continuous EEG can be described as the linear sum of (possibly) several overlapping event-related EEG responses. Depending on

194 the latencies of the neighboring events, these overlapping responses occur at different times $\tau$ relative to the current event (see Figure 2). That is, in the example in Figure 2, where the responses of two types of events, A and B overlap with each other, the observed continuous EEG at time point $t$ of the continuous EEG recording can be described as follows: 


$$
E E G_{t=25}=1 \beta_{A, 1}+0 \beta_{A, 2}+0 \beta_{A, 3}+0 \beta_{A, 4}+1 \beta_{A, 5}+0 \beta_{B, 1}+0 \beta_{B, 2}+0 \beta_{B, 3}+1 \beta_{B, 4}+0 \beta_{B, 5}
$$

In the example in Figure 2, the spontaneous EEG at time-point $t$ is modeled as the linear

200

201

202

203

204

205

206

207

208

209

210

211

212

213

214

215

216

217

218

219

220

221

222

223

sum of a to-be-estimated response to the first instance of event type A at local time $\tau=5$ (i.e. from the point of view of $E E G(t)$ this instance occurred 5 time samples before). Another response to the second instance of event type A at local time $\tau=0$ (i.e. this instance just occurred at $t$ ), and another response to the instance of event type B at local time $\tau=4$.

The necessary design matrix to implement this model, $X_{d c}$, will span the duration of the entire EEG recording. It can be generated from any design matrix $X$ by an algorithm we will call time expansion in the following. In this process, each predictor in the original design matrix will be expanded to several columns, which then code a number of "local" time points relative to the event onset. An example for a time-expanded design matrix is shown in Figure 2.

\section{Time expansion}

The process to create the time-expanded design matrix $X_{d c}$ is illustrated in Figure 2. In the following sections, we will describe the construction of $X_{d c}$ more formally.

Let $t$ be the time of the continuous EEG signal $y$, which keeps increasing throughout the experiment. $\tau$ is still the local time, that is the temporal distance of an EEG sample relative to an instance of event $e$. Let $i$ be the instance of one such event. $X_{i}$ is therefore the accompanying row of the design matrix $X$ which specifies the predictors for each event of type $e$. The design matrix $\mathrm{X}$ consists of multiple columns $c$, each representing one predictor (for which we want to estimate the accompanying $\beta$ ).

$X_{d c}$ can be constructed from multiple concatenated, time-shifted square diagonal matrices $G$ with size $\tau$ one for each instance of the event $e$. For the purpose of illustration, it is helpful to construct the design matrix first for just a single predictor and a single instance of a single type of event (e.g. a manual response). Afterwards, we will add multiple predictors, then multiple instances of a single event type and finally multiple different event types (e.g. stimuli and responses). 
225 The matrix $G_{c}$ for a single predictor, single type of event, and single instance of this event type is 226 square diagonal where the size is specified by the number of samples around the event instance 227 onsets to be taken into account:

$$
G_{c}=I x_{i, c}=\left[\begin{array}{cccc}
x_{i, c} & 0 & 0 & 0 \\
0 & x_{i, c} & 0 & 0 \\
0 & 0 & x_{i, c} & 0 \\
0 & 0 & 0 & x_{i, c}
\end{array}\right]
$$

230 It is a scaling of an identity matrix by the scalar $x_{i, c}$ which is a single entry of the design matrix $X$ 231 defining the predictor $c$ at the single instance $i$. In the case of a dummy-coded variable ( 0 or 1$)$ 232 we would get either a matrix full of zeros or the identity matrix; in case of a continuous predictor 233 we get a scalar matrix where the diagonal of $G_{i, c}$ contains the continuous predictor value.

\section{Multiple predictors, single instance, single event type}

In case of multiple predictors $c$, we generate multiple matrices $G_{i, c}$ and concatenate them to 237

$G^{*}=\left[G_{1} \ldots G_{c}\right]$. Therefore, a matrix with two predictors at the instance $i$ of an event $e$ could look 238 like:

$$
G^{*}=\left[G_{1} G_{2}\right]=\left[\begin{array}{cccccccc}
1 & 0 & 0 & 0 & 10 & 0 & 0 & 0 \\
0 & 1 & 0 & 0 & 0 & 10 & 0 & 0 \\
0 & 0 & 1 & 0 & 0 & 0 & 10 & 0 \\
0 & 0 & 0 & 1 & 0 & 0 & 0 & 10
\end{array}\right]
$$

Multiple predictors, multiple instances, single events type

242 In case of multiple instances of the same event, we have one $G{ }^{*}$ matrix for every instance. We 243 combine them into a large matrix $X_{d c}$ (where $d c$ stands for deconvolution) by inserting the $G^{*}$ 244 matrices into $X_{d c}$ around the time points (in continuous EEG time $t$ ) where the instance of the 245 event occurred. Because $\tau$ (and therefore $G^{*}$ ) is usually larger than the time distance between 246 two event instances, we insert rows of multiple $G^{*}$ matrices in an overlapping (summed) way. 247 Consequently, we model the same time point of the EEG by the combined rows of multiple $G^{*}$ 
248 matrices (Figure 2, Figure 3A). By solving the linear system with $X_{d c} \beta$ for $\beta$ we effectively

249 deconvolve the original signal.

250

251

252

253

254

255

256

257

258

259

260

261

262

263

264

265

266

267

268

269

270

271

272

273

274

\section{Multiple predictors, multiple instances, multiple events types}

We usually have multiple different types of events $e_{1}, e_{2} \ldots$. For each of these event types, we create one $X_{d c}^{e}$ matrix as described above. Each $X_{d c}^{e}$ matrix spans $t$ rows and thus, the continuous EEG signal. To get the final matrix $X_{d c}$ we simply concatenate them along the columns before the model inversion.

$$
X_{d c}=\left[X_{d c}^{1} \ldots X_{d c}^{e}\right]
$$

Similarly, if we wanted to include a continuous covariate spanning the whole duration of the continuous EEG signal (see Discussion), for example some feature of a continuous audio signal (e.g. Crosse, Di Liberto, Bednar, \& Lalor, 2016) we could simply concatenate it as an additional column to the design matrix.

The formula for the deconvolution model is then:

$$
\mu_{t}=X_{d c, t} \beta \text { with } y_{t} \sim \operatorname{normal}\left(\mu_{t}, \sigma\right)
$$

$\mu_{t}$ is the expected value of the continuous EEG signal $y_{t}$. This time-expanded linear model simultaneously fits all the parameters $\beta$ describing the deconvolved rERPs of interest. This comes at the cost of a very large size (number of samples $\times$ number of predictor columns). Fortunately, this matrix is also very sparse, containing mostly zeros, and can therefore be efficiently solved with modern sparse solvers. For further detail see the excellent tutorial reviews by Smith and Kutas (N. J. Smith \& Kutas, 2015a, 2015b).

\section{Modeling non-linear effects with spline regression}

Spline regression is a method to estimate non-linear relationships between a continuous predictor and an outcome. In the simple case of a single predictor it can be understood as a type of local smoother function. For detailed introduction to spline regression we recommend Harrell (2015) or Wood (2017). We will now outline how we can use spline predictors to model nonlinear effects within the linear regression framework. We follow the definition of a Generalized Additive Model (GAM) by Wood, (2017, p. 249-250):

Peer) reviewing PDF | (2018:12:33896:1:1:NEW 23 Aug 2019) 


$$
\mu_{i}=X_{i} \beta+\sum_{j} z_{j} f_{j}\left(x_{i}\right)
$$

276

The sum $\sum_{j} z_{j} f_{j}\left(x_{i}\right)$ represents a basis set with $j$ unknown parameters $z$ (analog to the $\beta$

277

278

280

281

282

283

284

285

286

287

288

289

290

291

292

293

294

295

296

297

298

299

$$
\mu_{i}=X_{i} \beta+z_{1} x^{1}+z_{2} x^{2}+z_{3} x^{3}
$$

However, due to several suboptimalities of the polynomial basis (e.g. Runge, 1901) we will make use of the cubic B-spline basis set instead. Spline regression is conceptually related to the better-known polynomial expansion, but instead of using polynomials, one uses locally bounded functions. In other words, whereas a polynomial ranges over the whole range of the continuous predictor, a B-spline is restricted to a local range.

This basis set is constructed using the De-Casteljau algorithm (De Casteljau, 1959) implemented by Bruno Luong (Luong, 2016). It is a basis set that is strictly local: Each basis function is non-zero only over a maximum of 5 other basis functions (for cubic splines; see Woods 2017, p. 204).

Multiple terms can be concatenated, resulting in a GAM:

$$
\mu_{i}=X_{i} \beta+\sum_{j} z_{j} f_{j}\left(x_{1 i}\right)+\sum_{j} z_{j} f_{j}\left(x_{2 i}\right)
$$

If interactions between two non-linear spline predictors are expected, we can also make use of two-dimensional splines:

$$
\mu_{i}=X_{i} \beta+\sum_{j} z_{j} f_{j}\left(x_{1 i}, x_{2 i}\right)
$$

In the unfold toolbox, 2D-splines are created based on the pairwise products between $z_{1, j}$ and $z_{2, j}$. Thus, a 2D spline between two spline-predictors with 10 spline-functions each would result in 100 parameters to be estimated.

The number of basis functions to use for each non-linear spline term are usually determined by either regularization or cross-validation. Cross-validation is difficult in the case of large EEG 
300 datasets (see also Discussion) and we therefore recommend to determine the number of splines

301 (and thus the flexibility of the non-linear predictors) prior to the analysis to avoid overfitting.

302 Using temporal basis functions

303 In the previous section, we made use of time-expansion to model the overlap. For this, we

304 used the so-called stick function approach (also referred to as FIR or dummy-coding). That is, each

305 time-point relative to an event (local time) was coded using a 1 or 0 (in case of dummy-coded

306 variables), resulting in a staircase patterns in the design matrix (cf. Figures 2 and 3A). However,

307 this approach is computationally expensive. Due to the high sampling rate of EEG (typically 200-

$3081000 \mathrm{~Hz}$ ), already a single second of estimated ERP response requires us to estimate 200-1000

309 coefficients per predictor. Therefore, some groups started to use other time basis sets to effectively

310 smooth the estimated regression-ERPs (e.g. Litvak et al., 2013; but see N. J. Smith \& Kutas, 311 2015b).

312 We will discuss two examples here: The time-Fourier set (Litvak et al., 2013; Spitzer et al., 313 2016) and - newly introduced in this paper - the time-spline set. In the time-spline set, adjacent

314 local time coefficients are effectively combined according to a spline set (Figure 3B). Splines are

315 a suitable basis function because EEG signals are smooth and values close in time have similar

316 values. The number of splines chosen here defines the amount of smoothing of the resulting

317 deconvolved ERP. The same principle holds for the time-Fourier set. Here we replace the stick-

318 function set with a truncated Fourier set (Figure 3C). Truncating the Fourier set at high frequencies

319 effectively removed high frequencies from the modeled ERP and can therefore be thought of as a

320 low-pass filter. A benefit of using (truncated) temporal basis functions rather than the simple stick

321 functions is that less unknown parameters need to be estimated, at the cost of temporal precision.

322 It is therefore possible that this results in numerical more stable solutions to the linear problem,

323 because, we are constraining the solution space. Low-pass filtering and downsampling of the data

324 followed by using the stick function deconvolution might result in better control of the spectral 325 properties. 
326

327

328

329

330

331

332

333

334

335

336

337

338

339

340

341

342

343

344

345

346

347

348

349

350

351

352

353

354

355

\section{Existing toolboxes}

To our knowledge, no existing toolbox supports non-linear, spline-based general additive modeling of EEG activity. Also, we were missing a toolbox that solely focuses on deconvolving signals and allowed for a simple specification of the model to be fitted, for example using the commonly used Wilkinson notation (as also used for example in $R$ ).

A few other existing EEG toolboxes allow for deconvolution analyses, but we found that each has their limitations. Plain linear modeling (including second-level analyses) can be performed using the LIMO toolbox (Pernet et al., 2011), but this toolbox does not support deconvolution analyses or spline regression. To our knowledge, five toolboxes support some form of deconvolution: SPM (Litvak et al., 2013; Penny, Friston, Ashburner, Kiebel, \& Nichols, 2006), the rERP extension for EEGLAB (Burns et al., 2013), pyrERP (N. J. Smith, 2013), mTRF (Crosse et al., 2016) and MNE (Gramfort et al., 2014). SPM allows for deconvolution of linear responses using Fourier temporal basis sets. However, in order to make use of these deconvolution functions, quite a bit of manual coding is needed. The rERP extension for EEGLAB and the pyrERP toolbox for Python both allow for estimation of linear models and deconvolution; however, both toolboxes appear not to be maintained anymore; rERP is currently non-functional (for current MATLAB versions) and no documentation is available for pyERP. The MNE toolbox is a general-purpose Python-based EEG processing toolbox that allows for both deconvolution and massive univariate modeling. It is actively maintained and some basic tutorials are available. The mTRF toolbox is a special type of deconvolution toolbox designed to be used with continuous predictors (e.g. auditory streams) that last over the whole continuous EEG recording (see Discussion).

\section{THE UNFOLD TOOLBOX}

In the following, we describe basic and advanced features available in the unfold toolbox and also give practical recommendations for problems that researchers might experience in the modeling process. Specifically, we describe how to (1) specify the model via Wilkinson formulas, (2) include nonlinear predictors via spline regression, (3) model the data with basis functions over time (e.g. a Fourier basis set), (4) impute missing data in the design matrix, (5) treat intervals of the continuous EEG containing EEG artifacts (e.g. from muscle activity or skin conductance changes), (5) specify alternative solvers (with regularization) that can solve even large models in a reasonable time and (6) run the same regression model both as a deconvolution model and also 
356 a mass multivariate model without deconvolution. Finally, we summarize options for (7)

357 visualizing and (8) exporting the results (Figure 4).

358 Data import

359 As a start, we need a data structure in EEGLAB format (Delorme \& Makeig, 2004) that 360 contains the continuous EEG data and event codes. In traditional EEG experiments, events will

361 typically be stimulus and response triggers, but many other types of events are also possible (e.g.,

362 voice onsets, the on- or offsets of eye or body movements etc.). In most cases, the EEG data entered

363 into the model should have already been corrected for biological and technical artifacts (e.g. ocular

364 artifacts, scalp muscle EMG, or power line noise), for example with independent component 365 analysis (ICA).

366

367

368

369

370

371

372

373

374

375

376

377

378

379

Specifying models using Wilkinson notation

We begin the modeling process by specifying the model formula and by generating the corresponding design matrix $\mathrm{X}$. In the unfold toolbox, models are specified using the intuitive Wilkinson notation (Wilkinson \& Rogers, 1973) also commonly used in $R$, the Matlab statistics toolbox, and python StatsModels. For example, for the hypothetical face/house experiment depicted in Figure 1, we might define the following model:

$\mathrm{EEG} \sim 1+\operatorname{cat}($ is_face $)+$ luminance

More generally, we can also specify more complex formulas, such as:

EEG $\sim 1+\operatorname{cat}($ predictor 1$)+$ predictor2 + spl (predictor 3$)$

Here, cat () specifies that the predictor1 should be dummy encoded as a categorical variable or factor rather than treated as a continuous variable. If a variable is already dummy-coded as $0 / 1$ it is not strictly necessary to add the cat ( ) command, but it is also possible to specify multilevel categorical variables. In contrast, predictor2 should be modeled as continuous linear covariate and predictor 3 as a nonlinear spline predictor. In the formula, a plus sign $(+)$ means 
380 that only the main effects will be modeled. Interactions between predictors are added by replacing

381 the + with a $*$ or a $:$, depending on whether all main effects and interactions should be modeled

$382(*)$, or only the interactions (:). In unfold, the type of coding (dummy/treatment/reference or

383 effect/contrast/sum coding) can be selected. If the default treatment coding is used, the predictors

384 will represent the difference to the intercept term (coded by the 1). The reference level of the

385 categorical variable and the ordering of the levels is determined alphabetically or can be specified

386 by the user manually. The toolbox also allows to specify different formulas for different events.

387 For example, stimulus onset events can have a different (e.g. more complex) formula than manual

388 response events.

389 Once the formula is defined, the design matrix $X$ is time-expanded to $\mathrm{X}_{d c}$ and now spans the 390 duration of the entire EEG recording. Subsequently, for each channel, the equation $391\left(\mathrm{EEG}=\mathrm{X}_{\mathrm{dc}} * b+\mathrm{e}\right)$ is solved for " $\mathrm{b}$ ", the betas, which correspond to subject-level rERP 392 waveforms. For example, in the model above, for which we used treatment coding, the intercept 393 term would correspond to the group-average ERP. The other betas, such as those for 394 cat (is_face), will capture the partial effect of that particular predictor, corresponding to a 395 difference wave in traditional ERPs (here: face-ERP minus house-ERP).

396 In the same linear model, we can simultaneously model brain responses evoked by other 397 experimental events, such as button presses. Each of these other event types can be modeled by its 398 own formula. In our face/house example, we would want to model the response-related ERP that 399 is elicited by the button press at the end of the trial, because this ERP will otherwise overlap to a 400 varying degree with the stimulus-ERP. We do this by defining an additional simple intercept model 401 for all button press events. In this way, the ERP evoked by button presses will be removed from 402 the estimation of the stimulus ERPs. The complete model would then be: $\mathrm{EEG}_{\mathrm{fix}} \sim 1+\operatorname{cat}($ is_face) $+\operatorname{spl}($ luminance,5) $\quad$ for stimulus onset events $\}$ $E E G_{\text {button }} \sim 1$ ffor manual button press events\}

Spline regression to model (nonlinear) predictors

As explained earlier, many influences on the EEG are not strictly linear. In addition to linear terms, one can therefore use cubic B-splines to perform spline regression, an approach commonly 
408 summarized under the name GAM (generalized additive modeling). An illustration of this 409 approach is provided in Figure 5. In the unfold toolbox, spline regression can be performed by 410 adding spl() around the predictor name, as for predictor 3 in the formula above, which 411 specifies a model using 5 B-splines instead of a continuous linear predictor. We can model 412 covariates as non-linear predictors:

$$
\mathrm{EEG} \sim 1+\operatorname{spl}(\mathrm{A}, 5)+2 \mathrm{dspl}(\mathrm{B}, \mathrm{C}, 5)+\operatorname{Circspl}(\mathrm{D}, 5,0,360)
$$

With this formula, the effect "A" would be modeled by a basis set consisting of ten splines. 415 We would also fit a 2D spline between continuous variable B and $\mathrm{C}$ with ten splines each. In 416 addition, we would fit a circular spline based on covariate $\mathrm{D}$ using ten splines with the limits $0^{\circ}$

and $360^{\circ}$ being the wrapping point of the circular spline.

In unfold, three spline functions are already implemented. For B-splines we use the de Casteljau algorithm implemented by Bruno Luong. For interactions between spline-modeled covariates, first the default spline function is used on each predictor to generate $n$ splines. Then the resulting vectors are elementwise multiplied with each other, generating $n^{2}$ final predictors. For cyclical predictors such as the angle of a saccadic eye movement (which ranges e.g. from 0 to $2 \pi$ ), it is possibly to use cyclical B-splines, as explained above. These are implemented based on code from patsy, a python statistical package (https://patsy.readthedocs.io) which follows an algorithm described in Woods (Wood, 2017, pp. 201-205). For maximal flexibility, we also allow the user to define custom spline functions. This would also allow to implement other basis sets, for example polynomial expansion.

In our default B-spline implementation, the so-called knots define the peaks of the cubic splines, except for the boundaries (i.e. extremes) of the covariate (see Figure 5B). The resulting number of splines are given by the number of knots minus 4 ( 4 due to the cubic nature, see Wood 2017 p. 204), but the user only has to specify the number of basis functions, not the exact number of knots. The placement of knots (and therefore the number of splines) are critical parameters to appropriately model the predictor and to avoid over- or underfitting the data. The toolbox's default 
434 knot placement is on the quantiles of the predictor, which will increase resolution of the splines in 435 areas where there is a lot of data points and offer stronger smoothing in other areas, where predictor 436 values are sparser (similar to Harrell, 2015, p. 26). This can be changed by users who want to use 437 a custom sequence of knots. Generalized cross-validation or penalized regression could be used to 438 narrow down the number of knots to be used but is computational expensive and currently not 439 supported in unfold.

\section{Using time basis functions}

Temporal basis functions were introduced earlier. The stick-function approach, as also illustrated in Figures 2 and $3 \mathrm{~A}$ is the default option in unfold. As alternatives, it is also possible to employ either a Fourier basis set or a set of temporal spline function. For example, for the timeexpansion step, Litvak and colleagues (Litvak et al., 2013; Spitzer et al., 2016) used a Fourier basis sets instead of stick-functions. Figure 6 compares simulation results for stick functions with those obtained with a Fourier basis set and a spline basis set in terms of the spectral components and the resulting filter artifacts. At this point, more simulation studies are needed to understand the effects of temporal basis sets on EEG data. We therefore follow the recommendation of Smith \& Kutas (N. J. Smith \& Kutas, 2015b) to use stick-functions for now.

Imputation of missing values

If a predictor has a missing value in massive univariate regression models, it is typically necessary to remove the whole trial. One workaround for this practical problem is to impute (i.e. interpolate) the value of missing predictors. In the deconvolution case, imputation is even more important for a reliable model fit, because if a whole event is removed, then overlapping activity from this event with that of the neighboring events would not be accounted for. In unfold we information, or imputation by the marginal, mean, or median values of the other events. 


\section{Dealing with EEG artifacts}

Linear deconvolution needs to be performed on continuous, rather than epoched data. This creates challenges with regard to the treatment of intervals that contain EEG artifacts. The way to handle artifacts in a linear deconvolution model is therefore to detect - but not to remove - the intervals containing artifacts in the continuous dataset. For these contaminated intervals, the timeexpanded design matrix $\left(\mathrm{X}_{\mathrm{dc}}\right)$ is then blanked out, that is, filled with zeros, so that the artifacts do not affect the model estimation (N. J. Smith \& Kutas, 2015b). If the data would have been cleaned prior to the time-expansion step, then events might have been removed that would overlap with clean data segments.

Of course, this requires the researcher to use methods for artifact correction that can be applied to continuous rather than segmented data (such as ICA). Similarly, we need methods that can detect residual artifacts in the continuous rather than epoched EEG. One example would be a peak-to-peak voltage threshold that is applied within a moving time window that is shifted stepby-step across the entire recording. Whenever the peak-to-peak voltage within the window exceeds a given threshold, the corresponding interval would then be blanked out in the design matrix. Detecting artifacts in the continuous rather than segmented EEG also has some small additional benefit, because if the data of a trial is only partially contaminated, the clean parts can still enter the model estimation (N. J. Smith \& Kutas, 2015b).

The unfold toolbox includes a function to remove artifactual intervals from the design matrix before fitting the model. In addition, we offer basic functionality, adapted from ERPLAB (LopezCalderon \& Luck, 2014), to detect artifacts in the continuous EEG.

\section{Multiple solvers: LSMR \& glmnet}

Solving for the betas is not always easy in such a large model. We offer several algorithms to solve for the parameters. The currently recommended one is LSMR (Fong \& Saunders, 2011), an iterative algorithm for sparse least-squares problems. This algorithm allows to use very large design matrices as long as they are sparse (i.e. contain mostly zeroes) which is usually the case if one uses time-expansion based on stick-functions (cf. Figures 2 and $3 \mathrm{~A}$ ).

However, especially with data containing a high level of noise, the tradeoff between bias and variance (i.e. between under- and overfitting) might be suboptimal, meaning that the parameters 
488

489

490

491

492

493

494

495

496

497

498

499

500

501

502

503

504

505

506

507

508

509

510

estimated from the data might be only weakly predictive for held-out data (i.e. they show a high variance and tend to overfit the data). Regularization is one way to prevent overfitting of parameter estimates. In short, regularization introduces a penalty term for high beta values, effectively finding a trade-off between overfit and out-of-sample prediction. The unfold toolbox allows the user to specify alternative solvers that use regularization. In particular, we include the glmnetsolver (Qian, Hastie, Friedman, Tibshirani, \& Simon, 2013), which allows for ridge (L2-norm), lasso (L1, leads to sparse solutions) and elastic net regularization. The regularization parameter is automatically estimated using cross-validation but the elastic-net parameter (deciding between L1 and L2 norm) has to be specified manually. Procedures to regularize with linear deconvolution have recently been examined and validated by Kristensen et al. (2017). Effects of regularization on noisy data are also depicted in Figure 7, which compares deconvolution results for noisy simulated data with and without regularization. In this simulation we used strongly correlated predictors $(r=0.85)$, thereby increasing collinearity. Note that collinearity in itself is only a problem for very extreme cases; specifically, if the matrix becomes ill-conditioned, the parameter solution might be instable and the estimands will "explode". Regularization can help in this situation. As can be seen in Figure 7, the non-regularized estimates show strong variance (panel B and C), whereas the regularized estimates show strong bias (panel D and E), that is, the estimated effects are shrunk towards zero but simultaneously, the variance of the estimate over time is greatly reduced. At this point, it is not yet clear whether and what type of regularization should be used for the standard analysis of EEG data, but we provide different solvers in unfold to facilitate future work on this topic. Please also see Kristensen et al. (2017a) for more simulation work.

Spatial vs. temporal deconvolution

511 Many researchers use source reconstruction (e.g. MNE, LORETA) or blind source 512 separation methods (e.g. ICA) to try to isolate the signal contributions of individual neural sources. 513 In our framework, this can be understood as performing a spatial deconvolution of the signal that 514 addresses the problem of volume conduction. Nevertheless, the activity time courses of each neural 
515 source may still overlap in time, for example due to repeated stimulus presentations. To apply the

516 deconvolution, it does not matter whether the input time series consists of raw EEG signals, or 517 they are the result of spatial filtering (e.g. beamformer: Van Veen, Van Drongelen, Yuchtman, \&

518 Suzuki, 1997), blind source separation (Makeig, Bell, JungTzyy-Ping, \& Sejnowski, 1996;

519 Delorme, Palmer, Onton, Oostenveld, \& Makeig, 2012) or some other transformation (Cohen, 520 2017). To use our toolbox with other types of time series, the data has to be simply copied into the 521 EEG.data matrix in EEGLAB. For convenience, we also offer a flag ('ica', 'true'), which 522 allows the researcher to directly model ICA activations (stored in EEG.icaact), instead of the 523 raw EEG. While we can only speculate about this issue at this point, it seem likely that a prior 524 spatial decomposition of the data improves the performance and interpretability of the final, 525 spatially and temporally deconvolved signals (Burwell, Makeig, Iacono, \& Malone, 2019).

\section{Comparison to a Mass Univariate model (without deconvolution)}

527 The unfold toolbox offers the option to compute a mass univariate regression model on the 528 same data using the exact same model but without correction for overlap. In our experience, 529 running this model in addition to the linear deconvolution model can be helpful to understand the 530 impact of overlap on the results. However, with this function, unfold can also be used as a 531 standalone toolbox for Mass-Univariate modeling, for the (rare) cases in which an experiment does 532 not involve any overlapping activity (e.g. from small saccades; Dimigen, Valsecchi, Sommer, \& 533 Kliegl, 2009).

534 Visualization of results

535 unfold offers multiple inbuilt functions to visualize rERP results (Figure 8). We provide 536 functions for marginal plots over splines and continuous variables, and functions to evaluate 537 splines/continuous covariates at specific values. For the topographical output we make use of 538 functions from the EEGVIS toolbox (Ehinger, 2018).

539 Exporting the results

540 unfold focusses on two main things: linear deconvolution and (non)-linear modeling at the 541 single-subject level. In contrast, the toolbox itself does not offer functions for group-level statistics. 
542 However, the betas for each participant can be easily exported as plain text (.csv) or as different

543 MATLAB structures to apply statistics with other toolboxes. A tutorial to process unfold results 544 using group-level permutation tests with the TFCE-toolbox (Mensen \& Khatami, 2013) is 545 provided in the online documentation.

546 A minimal but complete analysis script

$547 \quad$ Figure 9 shows a complete analysis script for the hypothetical face/house experiment 548 introduced above (see Figure 1). As can be seen, it can be run with a few lines of code. 
551 In this section we validate the unfold toolbox based on (1) simulated data and (2) a real

552 dataset from a standard face recognition ERP experiment containing overlapping activities.

553 Simulated data

554 To create simulated data, we produced overlapped data using four different response shapes, 555 shown in the first column of Figure 10: (1) a boxcar function, (2) a Dirac delta function, (3) a 556 simulated auditory ERP (the same as used by Lütkenhöner, 2010), and (4) random pink noise. We 557 then simulated $5 \mathrm{~s}$ of continuous data, during which 18 experimental events happened. Intervals 558 between subsequent events were randomly drawn from a normal distribution $(M=0.25 \mathrm{~s}, S D=$ $5590.05 \mathrm{~s})$. Convolving the simulated responses with the randomly generated event latencies produced 560 the continuous overlapped signal depicted in the third column of Figure 10. The last column of 561 Figure 10 shows the non-overlapped responses recovered by unfold (orange lines). For 562 comparison, overlapped responses without deconvolution are plotted in dark red. As can be seen, 563 unfold recovered the original response in all cases. The data of Figure 1 were also simulated and 564 then analyzed using our toolbox. Together, these simulations show that unfold successfully 565 deconvolves heavily overlapping simulated signals.

Real data example

568 Finally, we will also analyze a real dataset from a single participant who performed a 569 standard ERP face discrimination experiment ${ }^{1}$. In this experiment, previously described in the 570 supplementary materials of Dimigen et al. (2009), participants were shown 120 different color 571 images of human faces $\left(7.5^{\circ} \times 8.5^{\circ}\right)$ with a happy, angry, or neutral expression. All participants 572 provided written informed consent forms before taking part in the study (at the time of data 573 collection, there was no requirement to obtain institutional review board approval for individual

${ }^{1}$ The same example data was also analyzed in our accompanying paper (Dimigen \& Ehinger, 2018), but with a different focus. Further details on this dataset are given in Dimigen (2009) or Dimigen \& Ehinger (2018). 
574 ERP experiments that used standard procedures at the Department of Psychology at Humboldt 575 University). The participants' task was to categorize the emotion of the presented face as quickly 576 as possible using three response buttons, operated with the index, middle, and ring finger of the 577 right hand. Each stimulus was presented for $1350 \mathrm{~ms}$. The participant's mean RT was $836 \mathrm{~ms}$.

578 Although a central fixation cross was presented prior to each trial and participants were 579 instructed to avoid eye movements during the task, concurrent video-based eye-tracking revealed 580 that participants executed at least one involuntary (micro)saccades during the vast majority of trials 581 (see also Dimigen et al., 2009; Yuval-Greenberg, Tomer, Keren, Nelken, \& Deouell, 2008). For 582 the participant analyzed here, the median amplitude of these small saccades was $0.6^{\circ}$ and most 583 were aimed at the mouth region of the presented faces, which was most informative for the emotion 584 discrimination task.

This means that our stimulus-locked ERPs are contaminated with two other processes: visually-evoked potentials (lambda waves) generated by the retinal image motion produced by the (micro)saccades (Gaarder, Krauskopf, Graf, Kropfl, \& Armington, 1964; Dimigen et al., 2009) and motor processes related to preparing and executing the finger movement.

To disentangle these potentials with unfold, we specified three events: Stimulus onset, saccade onset, and button press. For this simple demonstration, we modeled both stimulus onsets and button press events using only an intercept term $(\mathrm{y} \sim 1)$, that is regardless of emotion. For the saccade onsets, we included both an intercept as well as saccade amplitude as a continuous predictor, because larger saccades are followed by larger lambda waves (e.g. Gaarder et al., 1964; Dimigen et al., 2009). Because this relationship is non-linear (e.g. Sangita Dandekar, Ding, Privitera, Carney, \& Klein, 2012) we used a set of 5 splines in the formula, $y \sim 1+\operatorname{spl}$ (saccade_amplitude, 5). Brain responses were modeled in the time window from $-1.5 \mathrm{~s}$ to $1 \mathrm{~s}$ around each event. Before fitting the model, we removed all intervals from the design matrix in which the recorded activity at any channels differed by $>250 \mu \mathrm{V}$ within a $2 \mathrm{~s}$.

Figure 11 presents the results for occipital electrode $\mathrm{Oz}$ and the signal both without (in red) and without (blue) the modeling and removal of overlapping activity. The large effect of overlapping activity can be clearly seen in the averaged ERP waveforms (top row in panels C, D,

603 and E). In the corresponding panels below that, we see the color-coded single trial activity 
604 (erpimages), in which segments time-locked to one type of event (e.g. stimulus onset) were sorted

605 by the latency of the temporally adjacent event (e.g. saccade onset). These panels clearly show the 606 overlapping activity and how it was successfully removed by the deconvolution. In particular, we 607 wish to highlight the substantial effect of overlap correction on the shape of both the stimulus608 onset ERP (elicited by the faces) and the response-related ERP (elicited by the button press), 609 despite the fact that average RT was relatively long (>800 ms) in this task. Microsaccades have 610 an additional distorting effect (Dimigen et al., 2009). We can therefore easily imagine how without 611 any overlap correction, differences in mean RT and microsaccade occurrence between condition 612 will create spurious condition effects in the stimulus-ERP. A more complex application where we 613 correct for similar spurious effects in a natural reading EEG experiment with 48 participants is 614 found in Dimigen \& Ehinger (2019). The data and code to reproduce figure 11 can be found under 615 https://osf.io/wbz7x/.

\section{DISCUSSION}

617 Human behavior in natural environments is characterized by complex motor actions and 618 quasi-continuous, multisensory stimulation. Brain signals recorded under such conditions are 619 characterized by overlapping activity evoked by different processes and typically also influenced 620 by a host of confounding variables that are difficult or impossible to orthogonalize under quasi621 experimental conditions. However, even in traditional, highly controlled laboratory experiments, 622 it is often unrealistic to match all stimulus properties between conditions, in particular if the stimuli 623 are high-dimensional, such as words (e.g. word length, lexical frequency, orthographic 624 neighborhood size, semantic richness, number of meanings etc.) or faces (e.g., luminance, contrast, 625 power spectrum, size, gender, age, facial expression, familiarity etc.). In addition, as we 626 demonstrate here, even simple EEG experiments often contain overlapping neural responses from 627 multiple different processes such as stimulus onsets, eye movements, or button presses. 628 Deconvolution modeling allows us to disentangle and isolate these different influences to improve 629 our understanding of the data.

630 In this article, we presented unfold, which deconvolves overlapping potentials and controls 631 for linear or non-linear influences of covariates on the EEG. In the following, we will discuss in 632 more detail the assumptions, possibilities, and existing limitations of this approach as well as 633 current and future applications. 


\section{Where can linear deconvolution be applied?}

635 Linear deconvolution can be applied to many types of paradigms and data. As shown above, 636 one application is to separate stimulus- and response-related components in traditional ERP studies 637 (see also Ouyang et al., 2011, 2015). Deconvolution is also particularly useful with complex ERP 638 designs that involve, for example, multimodal streams of visual, tactile, and auditory stimuli 639 (Spitzer et al., 2016). Deconvolution is also helpful in paradigms where it is problematic to find a 640 neutral interval to place a baseline, for example in experiments with fast tone sequences 641 (Lütkenhöner, 2010). In ERP research, the interval for baseline correction is usually placed 642 immediately before stimulus onset, but activity in this interval can vary systematically between 643 conditions due to overlapping activity, for example in self-paced paradigms (e.g. Ditman, 644 Holcomb, \& Kuperberg, 2007). This problem can be solved by deconvolving the signal first and 645 applying the baseline subtraction to the resulting isolated responses.

\section{Time-continuous covariates}

647 It is also possible to add time-continuous signals as predictors to the design matrix (Lalor, 648 Pearlmutter, Reilly, McDarby, \& Foxe, 2006; Crosse et al., 2016). Examples for continuous signals 649 that could be added as predictors include the luminance profile of a continuously flickering 650 stimulus (Lalor et al., 2006; VanRullen \& MacDonald, 2012), the sound envelope of an audio or 651 speech signal (with temporal lags to model the auditory temporal response function Crosse et al., 652 2016), the participants' gaze position or pupil size (from concurrent eye-tracking Dimigen \& 653 Ehinger, 2019), but also more abstract time series, such as predictions from a cognitive 654 computational model. Including time-continuous covariates such as gait-signals, movement 655 features, or environmental sounds could also improve the model fit in mobile EEG situations 656 (Ehinger et al., 2014; Gramann et al., 2014). The resulting betas of time-continuous covariates are 657 often named (multivariate) temporal response functions (mTRFs, but not consistently so, e.g. 658 Broderick, Anderson, Di Liberto, Crosse, \& Lalor, 2018 call FIR deconvolved betas TRFs). We 659 propose to follow this nomenclature but distinguish it from (stick-function-fitted) rERPs as we 660 have been using in this paper. Of course, one could combine both approaches and fit mTRFs and 661 rERPs simultaneously. 
662

663

664

665

666

667

668

669

670

671

672

673

674

675

676

677

678

679

680

681

682

683

684

685

686

687

688

689

690

\section{Underlying assumptions}

A fundamental assumption of traditional ERP averaging is that the shape of the underlying neural response is identical in all trials belonging to the same condition. Trials with short and long manual reaction times are therefore usually averaged together. Similarly, with linear deconvolution modeling, we assume that the brain response is the same for all events of a given type. However, like in traditional ERP analyses, we also assume that the neural response is independent of the interval between two subsequent events (e.g. the interval between a stimulus and a manual response). This is probably a simplification, since neural activity will likely differ between trials with a slow or fast reaction.

A related assumption concerns sequences of events: Processing one stimulus can change the processing of a following stimulus, for instance due to adaptation, priming, or other attentional effects. We want to note that if such sequential effects occur often enough in an experiment, they can be explicitly modeled; for example, on could add an additional predictors coding whether a stimulus is repeated or not or whether it occurred early or late in a sequence of stimuli. We hope that the unfold toolbox will facilitate the analysis of simulations on these issues and also propose to analyze experiments where temporal overlap is experimentally varied.

\section{Modeling nonlinear effects}

Nonlinear predictors can have considerable advantages over linear predictors. However, one issue that is currently unresolved is how to select an appropriate number of spline functions to model a nonlinear effect without under- or overfitting the data. While automatic selection methods exist (e.g. based on generalized cross-validation, Wood, 2017), their high computational cost of repeatedly deconvolving the data precluded us from using these techniques. In the current implementation of unfold, we assume the same number of splines are needed for all parts of the response. But it is possible, for example, that with a constant number of splines the baseline interval is overfitted, whereas the true response is underfitted. Therefore, algorithms to find smoothing parameters need to take into account that the amount of smoothing changes throughout the response. Choosing the correct number of splines that neither overfit nor underfit the data is an important question to resolve, and again, we hope that the unfold toolbox will facilitate future simulation studies, new algorithms, and new experiments on this issue. 
691

692 While all example analyses presented here were conducted in the time domain, it is also possible

693 to model and deconvolve overlapping time-frequency representations with unfold (see also Litvak

694 et al., 2013). One simple option is to enter the band-bass filtered EEG signal into the model (or

695 alternatively bandpass filter the estimated betas, but be aware of boundary filter artefacts). This

696

697

698

699

700

701

702

703

704

705

706

707

708

709

710

711

712

713

714

715

716

717

718

719 would model the evoked potentials. One could also estimate the instantaneous power for induced potentials. For instance Ossandón (2019) deconvolved the instantaneous power after band pass filtering the alpha band. But this assumes that the overlap is linear in power. Litvak et al. (2013) used an empirical approach based on model fit to decide between three possible power transformations (power, sqrt(power) and $\log ($ power)) and found slightly better model fit for the sqrt(power) transform. Alternatively, complex linear regression could be a solution (Hussin, Abdullah, \& Mohamed, 2010), but future work is needed here and we recommend more simulation studies prior to such time-frequency work. Choice of modelling parameters

In a traditional ERP analysis, the researcher has to set numerous analysis parameters, which will influence the final ERP results (e.g., filter settings, epoch length, baseline interval). Similarly, with deconvolution we have to make these and additional choices. Because the deconvolution approach is still in its infancy, there are not yet clearly established best-practices for all of the necessary settings. However, in the following, we discuss some basic and advanced parameters:

Basic settings/options

1) Sampling rate. Deconvolution can be applied to data recorded at any sampling rate, but some researchers have temporally downsampled their data (e.g. to $100 \mathrm{~Hz}$; Sassenhagen, 2018) before deconvolution. Aside from the obvious loss of temporal resolution that results from downsampling, we (anecdotally) have not observed a benefit for the stability of the estimation; in our experience, downsampling made the fitting process faster but not necessarily better.

2) Epoch size. The time limits for the deconvolution should be chosen so that the entire event-related response is modelled. For motor responses, such as saccades or button presses, it is therefore necessary to also include a sufficient number of timepoints before the event in order to capture (pre)motor potentials. 
720

721

722

723

724

725

726

727

728

729

730

731

732

733

734

735

736

737

738

739

740

741

742

743

744

745

746

747

748

749

Baseline correction. Baseline correction can be applied directly to the resulting betas $(\mathrm{N}$. J. Smith \& Kutas, 2015b). Whether baseline correction is always a good idea is still up to debate and in future work, it might be possible to include the baseline voltages directly into the GLM (Alday, 2019).

Advanced settings/options

3) Number of splines. The number of splines for a non-linear predictor is a difficult parameter to set, because it clearly depends on the underlying relation between predictor and ERP. Usually the number of splines is determined by penalized least squares, which is not supported by unfold. In our application to combined EEG and eye-tracking experiments (Dimigen \& Ehinger, 2019) we found that EEG effects like that of saccade size can be modelled by about 5 splines, whereas a much higher number of splines (e.g. 10) clearly overfitted the single subject data. In future implementations it might be possible to estimate the splines using penalized regression, mixed model fitting (Wood, 2017) or using cross-validation.

4) Placement of splines. The splines have to be placed along the range of the predictor variable. In unfold, the default placement of splines is on the $\left(\mathrm{N}_{\text {splines }}-2\right)$ percentiles of the predictor. Other placements are possible, and users can specify their own placements if necessary (Harrell, 2015, p. 26).

5) Regularization. Whether to use regularization at all and if so, what type of regularization to use, is currently not established. We recommend Kristensen et al. (2017) as a starting point.

6) Time basis used to generate $\mathrm{X}_{\mathrm{dc}}$. As described above, it is also possible to use a set of temporal basis functions (e.g. a Fourier set or set of temporal splines) to reduce the number of columns that is added to the design matrix in the time-expansion step. While unfold already implements this option, we follow for now the recommendation of Smith and Kutas (2015b) that simple stick functions (FIR) should be used for most applications.

\section{Outlook: Integration with linear mixed models}

In recent years, linear mixed-effects models (LMM, e.g. Gelman \& Hill, 2007) have been slowly superseding traditional models like repeated-measures ANOVA or the two-stage hierarchical approach used here. LMMs allow to model the hierarchical structure of single-subject 
750 and group-level data directly and have several other advantages, for example when analyzing

751 unbalanced designs (Baayen, Davidson, \& Bates, 2007; Kliegl, 2010). Combining LMMs with

752 linear deconvolution is theoretically possible. The main challenge is that one needs to fit all

753 continuous EEG datasets of all participants at the same time. Thus, currently, the high 754 computational cost of fitting such large models precludes us from taking advantage of mixed 755 models. Nevertheless, recent progress with similarly large models (Wood, Li, Shaddick, \& 756 Augustin, 2017) shows that the combination of LMMs with deconvolution modeling might be 757 computationally feasible in future implementations.

758 Other physiological signals

759 Finally, it is also possible to model other types of overlapping psychophysiological signals 760 with unfold, such as overlapping magnetic fields (MEG, Litvak et al., 2013), pupil dilations 761 (Wierda, van Rijn, Taatgen, \& Martens, 2012; Gagl, Hawelka, \& Hutzler, 2011) or skin 762 conductance responses (Bach, Flandin, Friston, \& Dolan, 2009).

763

764

765

766

767

768

769

770

771

772

\section{Conclusions}

In summary, unfold offers an integrated environment to analyze psychophysiological data influenced by overlapping responses, (non)linear covariates, or both. As we show above, this analysis strategy can be beneficial even in case of "traditional", highly-controlled ERP experiments. It also allows us to record EEG data under more natural situations, for example those with unconstrained eye movement behavior, which is typical for the emerging fields of virtual reality and mobile brain/body imaging studies. Applications of unfold to free viewing studies can be found in an accompanying paper (Dimigen \& Ehinger, 2019). The toolbox is freely available at http://www.unfoldtoolbox.org with tutorials and documentation. 
774

775

776

777

778

779

780

781

782

783

784

785

786

787

788

789

790

791

792

793

794

795

796

797

798

799

800

801

802

803

804

805

806

807

808

809

810

811

812

813

814

815

816
Alday, P. M. (2019). How much baseline correction do we need in ERP research? Extended GLM model can replace baseline correction while lifting its limits. Psychophysiology. https://doi.org/10.1111/psyp.13451

Amsel, B. D. (2011). Tracking real-time neural activation of conceptual knowledge using single-trial event-related potentials. Neuropsychologia, 49(5), 970-983. https://doi.org/10.1016/j.neuropsychologia.2011.01.003

Baayen, H. R., Davidson, D., \& Bates, D. M. (2007). Mixed-effect models R. Harald Baayen. Linguistics > Miscellaneous Papers, 59(4), ? https://doi.org/10.1016/j.jml.2007.12.005

Bach, D. R., Flandin, G., Friston, K. J., \& Dolan, R. J. (2009). Time-series analysis for rapid event-related skin conductance responses. Journal of Neuroscience Methods, 184(2), 224-234. https://doi.org/10.1016/j.jneumeth.2009.08.005

Bigdely-Shamlo, N., Touyran, J., Ojeda, A., Kothe, C., Mullen, T., \& Robbins, K. (2018). Automated EEG mega-analysis II: Cognitive aspects of event related features. BiorXiv. https://doi.org/https://doi.org/10.1101/411371

Broderick, M. P., Anderson, A. J., Di Liberto, G. M., Crosse, M. J., \& Lalor, E. C. (2018). Electrophysiological Correlates of Semantic Dissimilarity Reflect the Comprehension of Natural, Narrative Speech. Current Biology. https://doi.org/10.1016/j.cub.2018.01.080

Burns, M. D., Bigdely-Shamlo, N., Smith, N. J., Kreutz-Delgado, K., \& Makeig, S. (2013). Comparison of averaging and regression techniques for estimating Event Related Potentials. Conference Proceedings : ... Annual International Conference of the IEEE Engineering in Medicine and Biology Society. IEEE Engineering in Medicine and Biology Society. Conference, 2013, 1680-1683. https://doi.org/10.1109/EMBC.2013.6609841

Burwell, S. J., Makeig, S., Iacono, W. G., \& Malone, S. M. (2019). Reduced premovement positivity during the stimulus-response interval precedes errors: Using single-trial and regression ERPs to understand performance deficits in ADHD. Psychophysiology. https://doi.org/10.1111/psyp.13392

Coco, M., Nuthmann, A., \& Dimigen, O. (2018). Fixation-related brain activity during semantic integration of object-scene information. PsyArXiv. https://doi.org/10.31234/osf.io/efq48

Cohen, M. X. (2017). Comparison of linear spatial filters for identifying oscillatory activity in multichannel data. Journal of Neuroscience Methods, 278, 1-12. https://doi.org/10.1016/j.jneumeth.2016.12.016

Cornelissen, T., Sassenhagen, J., \& Võ, M. L.-H. (2019). Improving free-viewing fixation-related EEG potentials with continuoustime regression. Journal of Neuroscience Methods, 313, 77-94. https://doi.org/10.1016/j.jneumeth.2018.12.010

Crosse, M. J., Di Liberto, G. M., Bednar, A., \& Lalor, E. C. (2016). The Multivariate Temporal Response Function (mTRF) Toolbox: A MATLAB Toolbox for Relating Neural Signals to Continuous Stimuli. Frontiers in Human Neuroscience, 10. https://doi.org/10.3389/fnhum.2016.00604

Dale, A. M., \& Buckner, R. L. (1997). Selective averaging of rapidly presented individual trials using fMRI. Human Brain Mapping, 5(5), 329-340. https://doi.org/10.1002/(SICI)1097-0193(1997)5:5<329::AID-HBM1>3.0.CO;2-5

Dandekar, S., Privitera, C., Carney, T., \& Klein, S. A. (2012). Neural saccadic response estimation during natural viewing. Journal of Neurophysiology, 107(6), 1776-1790. https://doi.org/10.1152/jn.00237.2011

Dandekar, Sangita, Ding, J., Privitera, C., Carney, T., \& Klein, S. A. (2012). The Fixation and Saccade P3. PLoS ONE, 7(11). https://doi.org/10.1371/journal.pone.0048761

De Casteljau, P. (1959). Courbes à pôles. National Industrial Property Institute (France). https://doi.org/10.1.1.86.162

Delgado, R. E., \& Ozdamar, O. (2004). Deconvolution of evoked responses obtained at high stimulus rates. J Acoust Soc Am, 115(3), 1242-1251. https://doi.org/10.1121/1.1639327

Delorme, A., \& Makeig, S. (2004). EEGLAB: an open source toolbox for analysis of single-trial EEG dynamics including independent component analysis. Journal of Neuroscience Methods, 134(1), 9-21. https://doi.org/10.1016/j.jneumeth.2003.10.009

Delorme, A., Palmer, J., Onton, J., Oostenveld, R., \& Makeig, S. (2012). Independent EEG sources are dipolar. PLoS ONE, 7(2), e30135. https://doi.org/10.1371/journal.pone.0030135

PeerJ reviewing PDF | (2018:12:33896:1:1:NEW 23 Aug 2019) 
817 Dimigen, O., \& Ehinger, B. V. (2019). Analyzing combined eye-tracking/EEG experiments with (non)linear deconvolution models.

818 BioRxiv. https://doi.org/https://doi.org/10.1101/735530

819 Dimigen, O., Sommer, W., Hohlfeld, A., Jacobs, A. M., \& Kliegl, R. (2011). Coregistration of eye movements and EEG in natural

820

821

822

823 reading: Analyses and review. Journal of Experimental Psychology: General, 140(4), 552-572. https://doi.org/10.1037/a0023885

Dimigen, O., Valsecchi, M., Sommer, W., \& Kliegl, R. (2009). Human Microsaccade-Related Visual Brain Responses. Journal of Neuroscience, 29(39), 12321-12331. https://doi.org/10.1523/JNEUROSCI.0911-09.2009

824

825

Ditman, T., Holcomb, P. J., \& Kuperberg, G. R. (2007). An investigation of concurrent ERP and self-paced reading methodologies. Psychophysiology, 44(6), 927-935. https://doi.org/10.1111/j.1469-8986.2007.00593.x

826 Ehinger, B. V. (2018). EEGVIS Toolbox. Https://Github.Com/Behinger/Eegvis/. https://doi.org/10.5281/zenodo.1312813

827

828

829

830

831

832

833

Ehinger, B. V., Fischer, P., Gert, A. L., Kaufhold, L., Weber, F., Pipa, G., \& König, P. (2014). Kinesthetic and vestibular information modulate alpha activity during spatial navigation: a mobile EEG study. Frontiers in Human Neuroscience, 8. https://doi.org/10.3389/fnhum.2014.00071

Eysholdt, U., \& Schreiner, C. (1982). Maximum length sequences-a fast method for measuring brain-stem-evoked responses. Audiology, 21, 242-250.

Fong, D. C.-L., \& Saunders, M. (2011). LSMR: An Iterative Algorithm for Sparse Least-Squares Problems. SIAM Journal on Scientific Computing, 33(5), 2950-2971. https://doi.org/10.1137/10079687X

834

835

Frömer, R., Maier, M., \& Abdel Rahman, R. (2018). Group-Level EEG-Processing Pipeline for Flexible Single Trial-Based Analyses Including Linear Mixed Models. Frontiers in Neuroscience, 12. https://doi.org/10.3389/fnins.2018.00048

836

Gaarder, K., Krauskopf, J., Graf, V., Kropfl, W., \& Armington, J. C. (1964). Averaged Brain Activity Following Saccadic Eye Movement. Science, 146(3650), 1481-1483. https://doi.org/10.1126/science.146.3650.1481

838

839

Gagl, B., Hawelka, S., \& Hutzler, F. (2011). Systematic influence of gaze position on pupil size measurement: Analysis and correction. Behavior Research Methods, 43(4), 1171-1181. https://doi.org/10.3758/s13428-011-0109-5

840 Gelman, A., \& Hill, J. L. (2007). Data analysis using regression and multilevel/hierarchical models. Policy Analysis.

841 https://doi.org/10.2277/0521867061

842

843

844

845

Gramann, K., Jung, T.-P., Ferris, D. P., Lin, C.-T., \& Makeig, S. (2014). Toward a new cognitive neuroscience: modeling natural brain dynamics. Frontiers in Human Neuroscience, 8. https://doi.org/10.3389/fnhum.2014.00444

Gramfort, A., Luessi, M., Larson, E., Engemann, D. A., Strohmeier, D., Brodbeck, C., ... Hämäläinen, M. S. (2014). MNE software for processing MEG and EEG data. NeuroImage, 86, 446-460. https://doi.org/10.1016/j.neuroimage.2013.10.027

846

Halliday, A. (1982). The visual evoked potentials in healthy subjects. In Evoked potentials in clinical testing (pp. 57-113). London: Churghill Livingstone.

848

849

Hansen, J. C. (1983). Separation of overlapping waveforms having known temporal distributions. Journal of Neuroscience Methods, 9(2), 127-139. https://doi.org/10.1016/0165-0270(83)90126-7

850 Harrell, F. E. (2015). Multivariable Modeling Strategies. https://doi.org/10.1007/978-3-319-19425-7_4

851

852

853

854

Hauk, O., Davis, M. H., Ford, M., Pulvermüller, F., \& Marslen-Wilson, W. D. (2006). The time course of visual word recognition as revealed by linear regression analysis of ERP data. NeuroImage, 30(4), $1383-1400$. https://doi.org/10.1016/j.neuroimage.2005.11.048

Hussin, A. G., Abdullah, N. A., \& Mohamed, I. (2010). A complex linear regression model. Sains Malaysiana, 39(3), $491-494$.

Jewett, D. L., Caplovitz, G., Baird, B., Trumpis, M., Olson, M. P., \& Larson-Prior, L. J. (2004). The use of QSD (q-sequence deconvolution) to recover superposed, transient evoked-responses. Clinical Neurophysiology, 115(12), $2754-2775$. https://doi.org/10.1016/j.clinph.2004.06.014

Kliegl. (2010). Experimental effects and individual differences in linear mixed models: estimating the relationship between spatial, object, and attraction effects in visual attention. Frontiers in Psychology, 1(1). https://doi.org/10.3389/fpsyg.2010.00238 
860

861

862

863

864

865

866

867

868

869

870

871

872

873

874

875

876

877

878

879

880

881

882

883

884

885

886

887

888

889

890

891

892

893

894

895

896

897

898

899

900

901

902

903

Kristensen, E., Guerin-Dugué, A., \& Rivet, B. (2017). Regularization and a general linear model for event-related potential estimation. Behavior Research Methods, 49(6), 2255-2274. https://doi.org/10.3758/s13428-017-0856-Z

Kristensen, E., Rivet, B., \& Guérin-Dugué, A. (2017). Estimation of overlapped Eye Fixation Related Potentials: The General Linear Model, a more flexible framework than the ADJAR algorithm. Journal of Eye Movement Research, 10(1), 1-27. https://doi.org/10.16910/JEMR.10.1.7

Lalor, E. C., Pearlmutter, B. A., Reilly, R. B., McDarby, G., \& Foxe, J. J. (2006). The VESPA: A method for the rapid estimation of a visual evoked potential. NeuroImage, 32(4), 1549-1561. https://doi.org/10.1016/j.neuroimage.2006.05.054

Litvak, V., Jha, A., Flandin, G., \& Friston, K. (2013). Convolution models for induced electromagnetic responses. NeuroImage, 64(1), 388-398. https://doi.org/10.1016/j.neuroimage.2012.09.014

Lopez-Calderon, J., \& Luck, S. J. (2014). ERPLAB: an open-source toolbox for the analysis of event-related potentials. Frontiers in Human Neuroscience, 8. https://doi.org/10.3389/fnhum.2014.00213

Luong, B. (2016). Free-knot spline approximation. Retrieved from https://de.mathworks.com/matlabcentral/fileexchange/25872free-knot-spline-approximation

Lütkenhöner, B. (2010). Baseline correction of overlapping event-related responses using a linear deconvolution technique. NeuroImage, 52(1), 86-96. https://doi.org/10.1016/j.neuroimage.2010.03.053

Makeig, S., Bell, A. J., JungTzyy-Ping, \& Sejnowski, T. J. (1996). Independent Component Analysisof Electroencephalographic Data. Advances in Neural Information Processing Systems, (8), 145-151. https://doi.org/10.1109/ICOSP.2002.1180091

Marsh, R. R. (1992). Signal to Noise Constraints on maximum length sequence auditory Brain-Stem Responses. Ear and Hearing, 13(6), 396-400. https://doi.org/10.1097/00003446-199212000-00004

Mensen, A., \& Khatami, R. (2013). Advanced EEG analysis using threshold-free cluster-enhancement and non-parametric statistics. NeuroImage, 67, 111-118. https://doi.org/10.1016/j.neuroimage.2012.10.027

Nunez, P. L., \& Srinivasan, R. (2006). Electric Fields of the Brain: The neurophysics of EEG. Electric Fields of the Brain: The neurophysics of EEG. Oxford University Press. https://doi.org/10.1093/acprof:oso/9780195050387.001.0001

Oostenveld, R., Fries, P., Maris, E., \& Schoffelen, J.-M. (2011). FieldTrip: Open source software for advanced analysis of MEG, EEG, and invasive electrophysiological data. Computational Intelligence and Neuroscience, 2011, 1-10. https://doi.org/10.1155/2011/156869

Ossandón, J. P., König, P., \& Heed, T. (2019). Spatially modulated alpha-band activity does not mediate tactile remapping and fast overt orienting behavior. BioRxiv, 576850. https://doi.org/10.1101/576850

Ouyang, G., Herzmann, G., Zhou, C., \& Sommer, W. (2011). Residue iteration decomposition (RIDE): A new method to separate ERP components on the basis of latency variability in single trials. Psychophysiology, 48(12), $1631-1647$. https://doi.org/10.1111/j.1469-8986.2011.01269.x

Ouyang, G., Sommer, W., \& Zhou, C. (2015). A toolbox for residue iteration decomposition (RIDE)-A method for the decomposition, reconstruction, and single trial analysis of event related potentials. Journal of Neuroscience Methods, 250, 7-21. https://doi.org/10.1016/j.jneumeth.2014.10.009

Penny, W., Friston, K., Ashburner, J., Kiebel, S., \& Nichols, T. (2006). Statistical Parametric Mapping: The Analysis of Functional Brain Images: The Analysis of Functional Brain Images. Statistical Parametric Mapping The Analysis of Functional Brain Images (Vol. 8). https://doi.org/10.1016/B978-012372560-8/50002-4

Pernet, C. R., Chauveau, N., Gaspar, C., \& Rousselet, G. A. (2011). LIMO EEG: a toolbox for hierarchical LInear MOdeling of ElectroEncephaloGraphic data. Computational Intelligence and Neuroscience, 2011, 831409. https://doi.org/10.1155/2011/831409

Qian, J., Hastie, T., Friedman, J., Tibshirani, R., \& Simon, N. (2013). Glmnet for Matlab. URL Http://Www. Stanford. Edu/ Hastie/Glmnet_matlab. Retrieved from http://www.stanford.edu/ hastie/glmnet_matlab/

Runge, C. (1901). Über empirische Funktionen und die Interpolation zwischen äquidistanten Ordinaten. Zeitschrift Für Mathematik Und Physik, 46, 224-243.

Peer) reviewing PDF | (2018:12:33896:1:1:NEW 23 Aug 2019) 
904

905

906

907

908

909

910

911

912

913

914

915

916

917

918

919

920

921

922

923

924

925

926

927

928

929

930

931

932

933

934

935

936

937

938

939

940

941

942

943

944

Sassenhagen, J. (2018). How to analyse electrophysiological responses to naturalistic language with time-resolved multiple regression. Language, Cognition and Neuroscience, (0), 1-17. https://doi.org/10.1080/23273798.2018.1502458

Smith, N. J. (2013). pyrERP. Retrieved from https://github.com/njsmith/pyrerp/

Smith, N. J., \& Kutas, M. (2015a). Regression-based estimation of ERP waveforms: I. The rERP framework. Psychophysiology, 52(2), 157-168. https://doi.org/10.1111/psyp.12317

Smith, N. J., \& Kutas, M. (2015b). Regression-based estimation of ERP waveforms: II. Nonlinear effects, overlap correction, and practical considerations. Psychophysiology, 52(2), 169-181. https://doi.org/10.1111/psyp.12320

Smith, S. M., \& Nichols, T. E. (2009). Threshold-free cluster enhancement: Addressing problems of smoothing, threshold dependence and localisation in cluster inference. NeuroImage, 44(1), 83-98. https://doi.org/10.1016/j.neuroimage.2008.03.061

Spitzer, B., Blankenburg, F., \& Summerfield, C. (2016). Rhythmic gain control during supramodal integration of approximate number. NeuroImage, 129, 470-479. https://doi.org/10.1016/j.neuroimage.2015.12.024

Talsma, D., \& Woldorff, M. G. (2004). Methods for the Estimation and Removal of Artifacts and Overlap in ERP Waveforms. In T. Handy (Ed.), Event-related potentials: A methods handbook. (pp. 115-148). Cambridge, MA: MIT Press. https://doi.org/10.1212/01.wnl.0000217365.45426.9a

Van Humbeeck, N., Meghanathan, R. N., Wagemans, J., van Leeuwen, C., \& Nikolaev, A. R. (2018). Presaccadic EEG activity predicts visual saliency in free-viewing contour integration. Psychophysiology, e13267. https://doi.org/10.1111/psyp.13267

Van Veen, B. D., Van Drongelen, W., Yuchtman, M., \& Suzuki, A. (1997). Localization of brain electrical activity via linearly constrained minimum variance spatial filtering. IEEE Transactions on Biomedical Engineering, 44(9), 867-880. https://doi.org/10.1109/10.623056

VanRullen, R., \& MacDonald, J. S. P. (2012). Perceptual echoes at $10 \mathrm{~Hz}$ in the human brain. Current Biology, $22(11), 995-999$. https://doi.org/10.1016/j.cub.2012.03.050

Wang, T., Özdamar, Ö., Bohórquez, J., Shen, Q., \& Cheour, M. (2006). Wiener filter deconvolution of overlapping evoked potentials. Journal of Neuroscience Methods, 158(2), 260-270. https://doi.org/10.1016/j.jneumeth.2006.05.023

Wierda, S. M., van Rijn, H., Taatgen, N. A., \& Martens, S. (2012). Pupil dilation deconvolution reveals the dynamics of attention at high temporal resolution. Proceedings of the National Academy of Sciences, 109(22), 8456-8460. https://doi.org/10.1073/pnas.1201858109

Wilkinson, G. N., \& Rogers, C. E. (1973). Symbolic Description of Factorial Models for Analysis of Variance. Applied Statistics, 22(3), 392. https://doi.org/10.2307/2346786

Woldorff, M. G. (1993). Distortion of ERP averages due to overlap from temporally adjacent ERPs: Analysis and correction. Psychophysiology, 30(1), 98-119. https://doi.org/10.1111/j.1469-8986.1993.tb03209.x

Wood, S. N. (2017). Generalized additive models: an introduction with R. Texts in Statistical Science, xvii, 392 p. https://doi.org/10.1111/j.1541-0420.2007.00905_3.x

Wood, S. N., Li, Z., Shaddick, G., \& Augustin, N. H. (2017). Generalized Additive Models for Gigadata: Modeling the U.K. Black Smoke Network Daily Data. Journal of the American Statistical Association, 112(519), 1199-1210. https://doi.org/10.1080/01621459.2016.1195744

Yuval-Greenberg, S., Tomer, O., Keren, A. S., Nelken, I., \& Deouell, L. Y. (2008). Transient Induced Gamma-Band Response in EEG as a Manifestation of Miniature Saccades. Neuron, 58(3), 429-441. https://doi.org/10.1016/j.neuron.2008.03.027

Zhang, J. (1998). Decomposing stimulus and response component waveforms in ERP. Journal of Neuroscience Methods, 80(1), 49-63. https://doi.org/10.1016/S0165-0270(97)00194-5

Peer] reviewing PDF | (2018:12:33896:1:1:NEW 23 Aug 2019) 


\section{Figure 1}

A hypothetical simple ERP experiment with overlapping responses and a non-linear covariate

(A) A hypothetical simple ERP experiment with overlapping responses and a non-linear covariate. Data in this figure was simulated and then modeled with unfold. Participants saw pictures of faces or house and categorized them with a button press. (B) A short interval of the recorded EEG. Every stimulus onset and every button press elicits a brain response (isolated responses). However, because brain responses to the stimulus overlap with that to the response, we can only observe the sum of the overlapping responses in the EEG (upper row). (C) Because humans are experts for faces, we assume here that they reacted faster to faces than houses, meaning that the overlap with the preceding stimulus-onset ERP is larger in the face than house condition. (D) Furthermore, we assume that faces and house stimuli were not perfectly matched in terms of all other stimulus properties (e.g. spectrum, size, shape). For this example, let us simply assume that they differed in mean luminance. (E) The N170 component of the fERP is typically larger for faces than houses. In addition, however, the higher luminance alone increases the amplitude of the visual P1 component of the ERP. Because luminance is slightly higher for faces and houses, this will result in a spurious condition difference. (F) Average ERP for faces and houses, without deconvolution modeling. In addition to the genuine N170 effect (larger N170 for faces), we can see various spurious differences, caused by overlapping responses and the luminance difference. (G) Linear deconvolution corrects for the effects of overlapping potentials. (H) To also remove the confounding luminance effect, we need to also include this predictor in the model. Now we are able to only recover the true N170 effect without confounds (a similar figure was used in Dimigen \& Ehinger, 2019). 
A

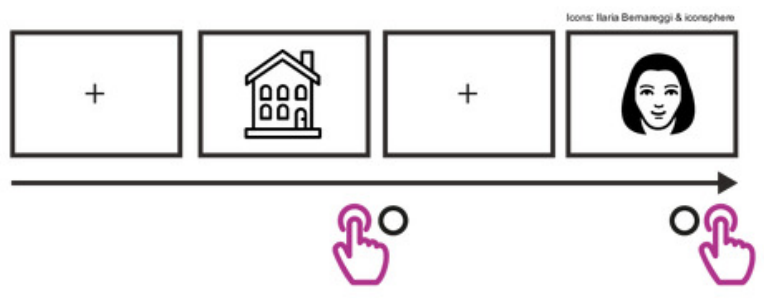

B

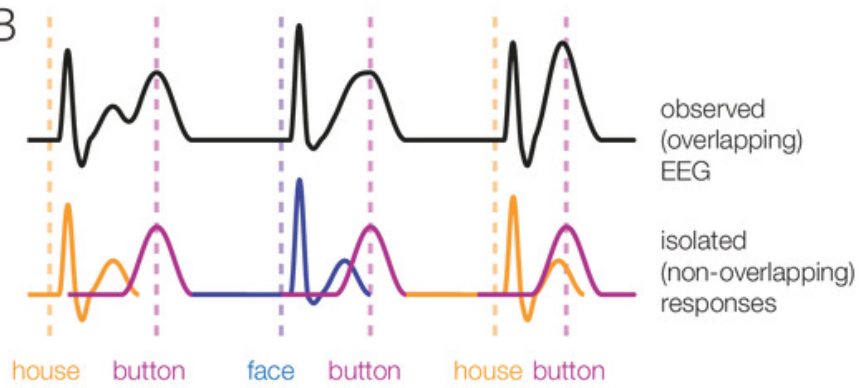

C

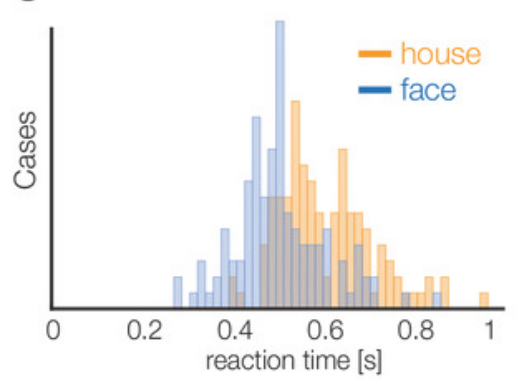

F

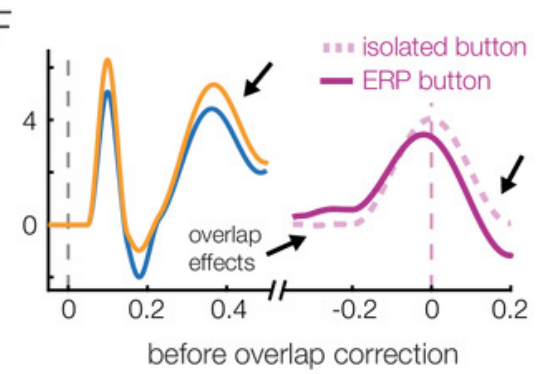

$\mathrm{D}$

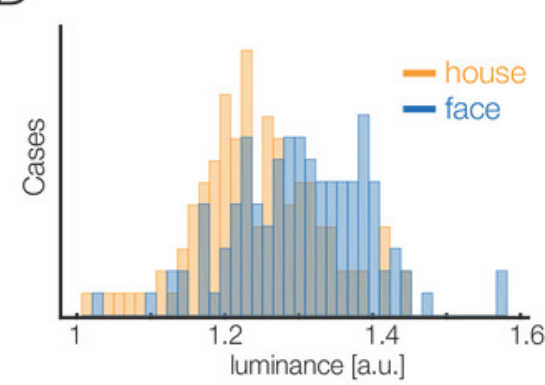

E

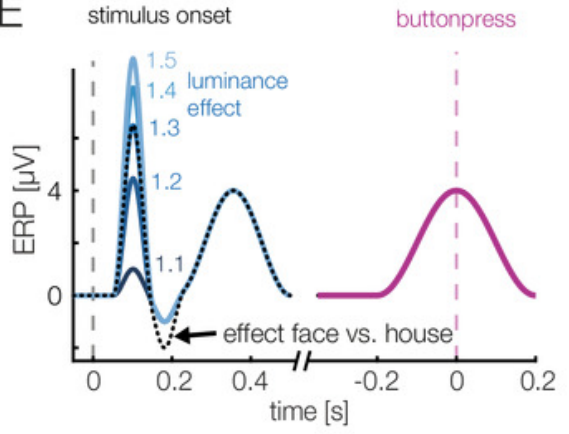

G

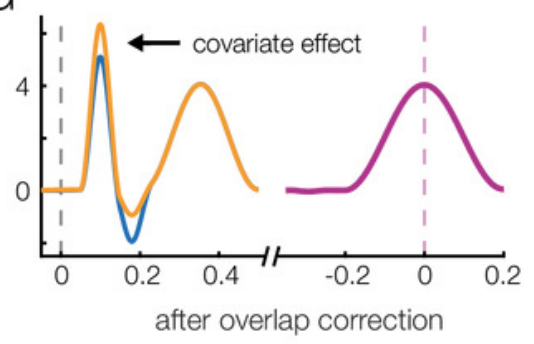

$\mathrm{H}$

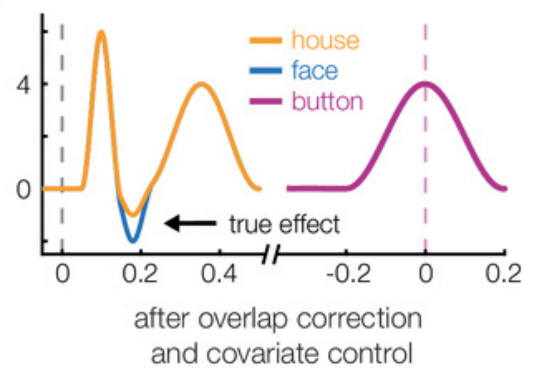




\section{Figure 2}

Linear Deconvolution by timeexpansion

Linear deconvolution explains the continuous (toy) EEG signal within a single regression model. Specifically, we want to estimate the response (betas) evoked by each event so that together, they best explain the observed EEG. For this purpose, we create a time-expanded version of the design matrix $(\mathrm{Xdc})$ in which a number of time points around each event (here: only 5 points) are added as predictors. We then solve the model for $b$, the betas. For instance, in the model above, sample number 25 of the continuous EEG recording can be explained by the sum of responses to three different experimental events: the response to a first event of type " $A$ " (at time point 5 after that event), by the response to an event of type "B" (at time 4 after that event) and by a second occurrence of an event of type " $A$ " (at time 1 after that event). Because the sequences of events and their temporal distances vary throughout the experiment, it is possible to find a unique solution for the betas that best explains the measured EEG signal. These betas, or "regression-ERPs" can then be plotted and analyzed like conventional ERP waveforms. Figure adapted from Dimigen \& Ehinger, 2019 (with permission). 


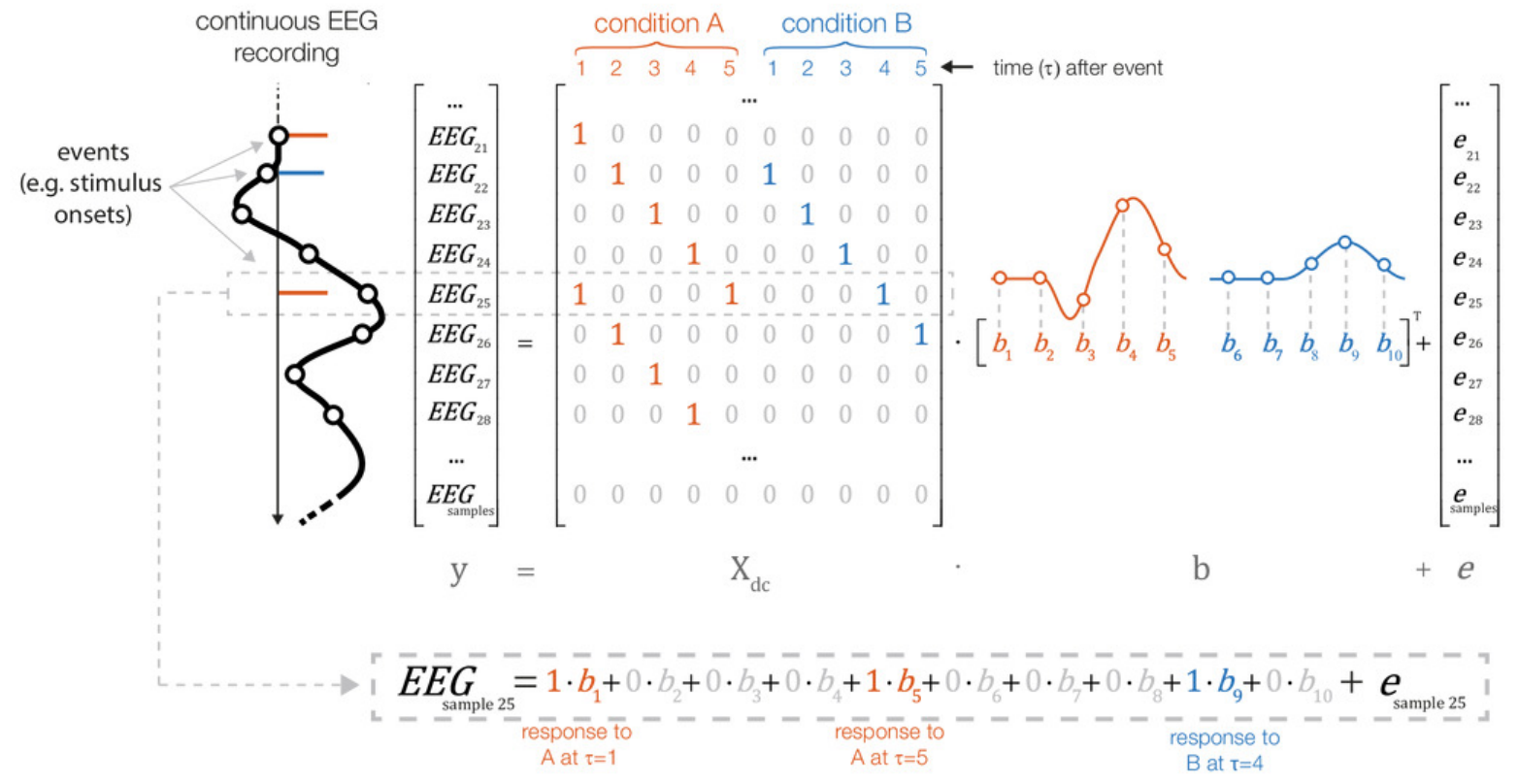




\section{Figure 3}

Temporal basis functions

Overview over different temporal basis functions. The expanded design matrix Xdc is plotted, the $y$-axis represents time and the $x$-axis shows all time-expanded predictors in the model. In unfold, three methods are available for time expansion: (A) Stick-functions. Here, each modeled time point relative to the event is represented by a unique predictor. (B) Timesplines allow neighboring time points to smooth themselves. This generally results in less predictors than the stick function set. (C) Truncated time-Fourier set: It is also possible to use a Fourier basis. By omitting high frequencies from the Fourier-set, the data are effectively low-pass filtered during the deconvolution process (see also Figure 6).
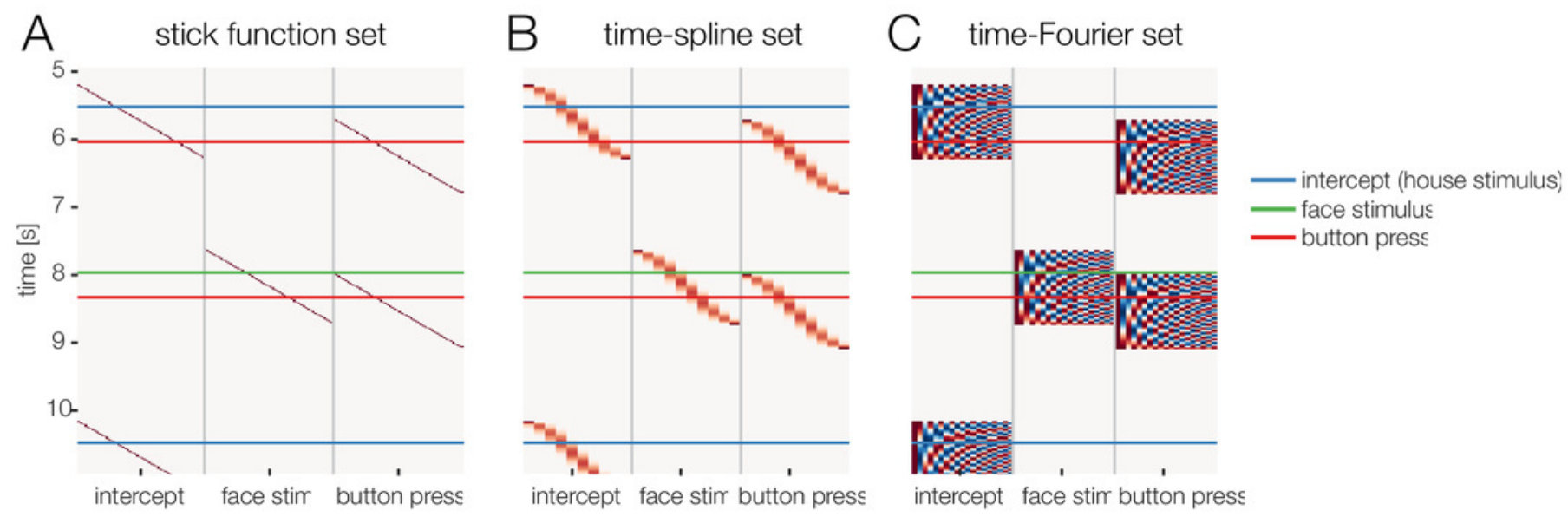


\section{Figure 4}

Overview over typical analysis steps with unfold.

The first step is to load a continuous EEG dataset into EEGLAB. This dataset should already contain event markers (e.g. for stimulus onsets, button presses etc.). Afterwards there are four main analysis steps, that can be executed with a few lines of code (see also Box 1). These steps, highlighted in blue, are: (1) Define the model formula and let unfold generate the design matrix, (2) time-expand this design matrix, (3) solve the model to obtain the betas (i.e. rERPs), and (4) convert the betas into a convenient format for plotting and statistics. The right column lists several inbuilt plotting functions to visualize intermediate analysis steps or to plot the results (see also Figure 8). 


\section{Analysis pipeline}

\section{Visualization functions}

Continuous EEG with events

Specify model \& generate design matrix
uf_designmat () $\rightarrow \begin{gathered}\text { Inspect events in data } \\ \text { uf_plotEventCorrmat () } \\ \text { uf_plotEventHistogram () } \\ \text { Pemove artifacts in continuous EEG } \\ \text { uf continuousArtifactexclude () } \\ \text { uf_plotDesignmat () }\end{gathered}$

Time-expand design matrix

uf_timeexpandDesignmat ()

Plot expanded design matrix uf_plotDesignmat ()

Solve regression model $(\mathrm{y}=\mathrm{Xb}+\mathrm{e})$

uf glmfit()

Repeat analysis without deconvolution uf epoch () uf_glmfit_nodc ( )

\section{Extract betas (\& apply time-basis) uf_condense ()}

Evaluate continuous or spline predictors at specific values uf_predictContinuous ()

Add marginal effects uf addmarginal ()

Export betas uf unfold2csv ( )

Second-level (group) statistics (e.g. TFCE toolbox)
Visualize rERPs

\section{Plot waveforms} uf_plotParam ()

Plot waveforms (cont. predictors) uf_plotParam2d()

Plot topographies uf_plotParamTopo () 


\section{Figure 5}

Modeling a nonlinear relationship with a set of spline functions.

(A) Example of a non-linear relationship between a predictor (e.g. stimulus luminance) and a dependent variable (e.g. EEG amplitude). A linear function (black line) does not fit the data well. We will follow one luminance value (dashed line) at which the linear function is evaluated (red dot). (B) Instead of a linear fit, we define a set of overlapping spline functions which are distributed across the range of the predictor. In this example, we are using a set of six b-splines. For our luminance value, we receive six new predictor values. Only three of them are non-zero. (C) We weight each spline with its respective estimated beta value. To predict the dependent variable (EEG amplitude) at our luminance value (dashed line), we sum up the weighted spline functions (red dots). Because the splines are overlapping, this produces a smooth, non-linear fit to the observed data.

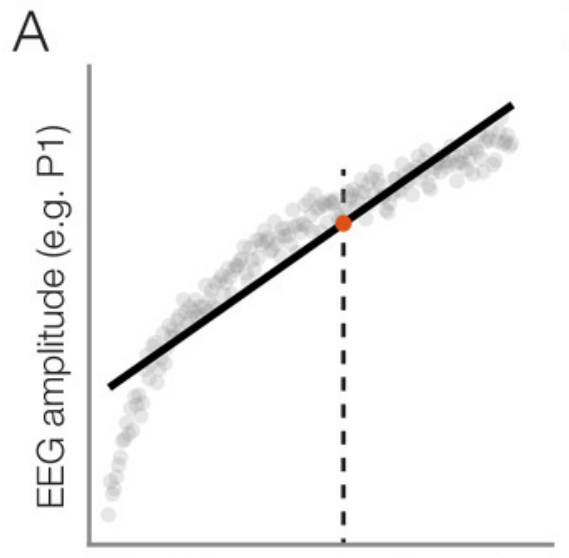

Predictor (e.g. luminance)

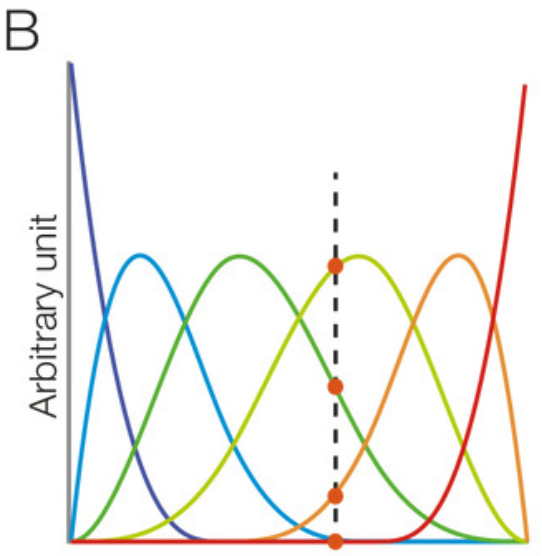

Predictor (e.g. luminance)

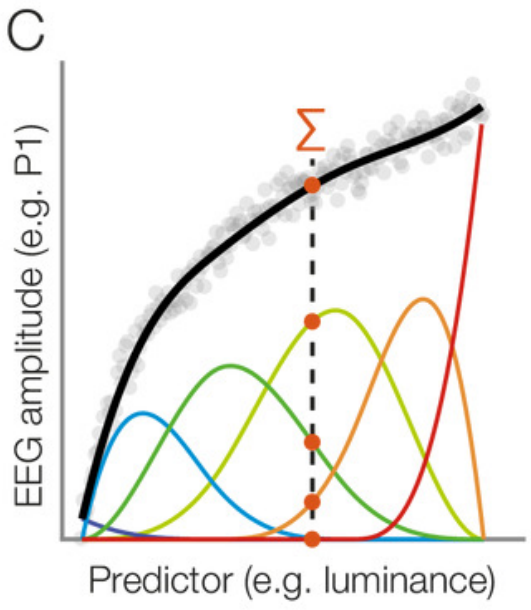




\section{Figure 6}

Using temporal basis functions

Effect of using different time basis functions on the recovery of the original signal using deconvolution. Panels A, B, and C show three different example signals without deconvolution (in black) and with convolution using different methods for the time-expansion (stick, Fourier, spline). We zero-padded the original signal to be able to show boundary artifacts. For the analysis we used 45 time-splines and in order to keep the number of parameters equivalent, the first 22 cosine and sine functions of the Fourier set. The smoothing effects of using a time-basis set can be best seen in the difference between the blue curve and the orange/red curves in panel $\mathrm{D}$. Artifacts introduced due to the time-basis set are highlighted with arrows and can be seen best in panels $E$ and F. Note that in the case of realistic EEG data, the signal is typically smooth, meaning that ripples like in panel $E$ rarely occur. (G) The impulse response spectrum of the different smoothers. Clearly, the Fourier-set filters better than the splines, but splines allow for a sparser description of the data and could benefit in the fitting stage. 


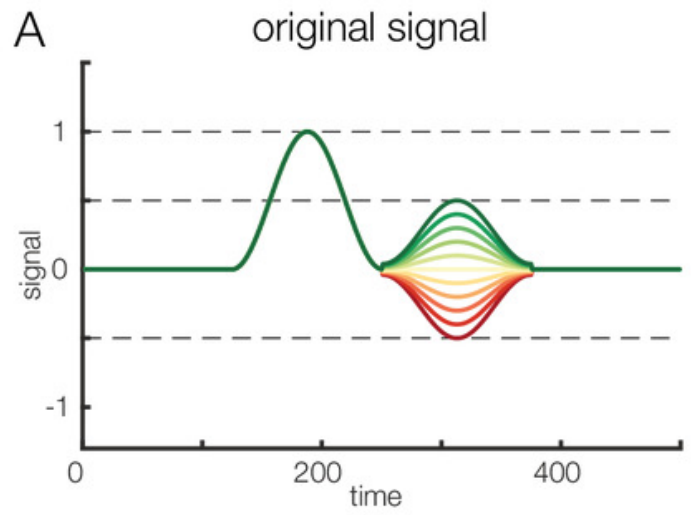

ordinary least squares

$\mathrm{B}$
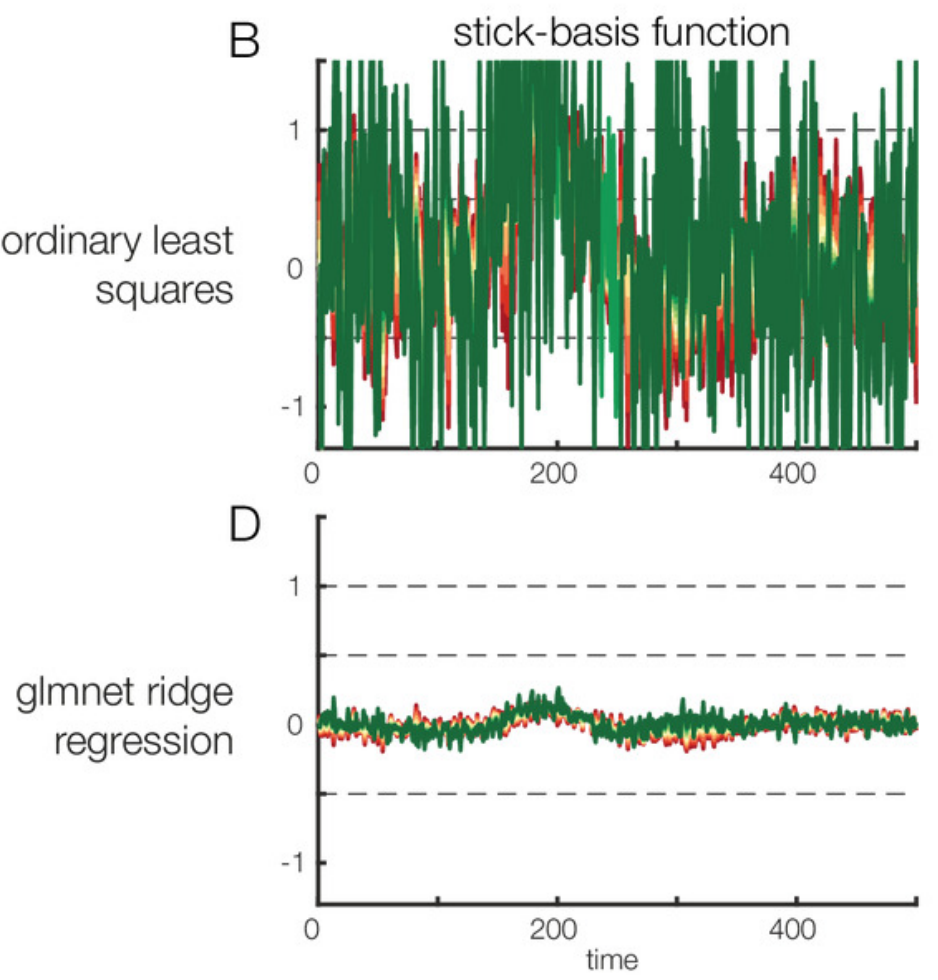

C spline-basis function

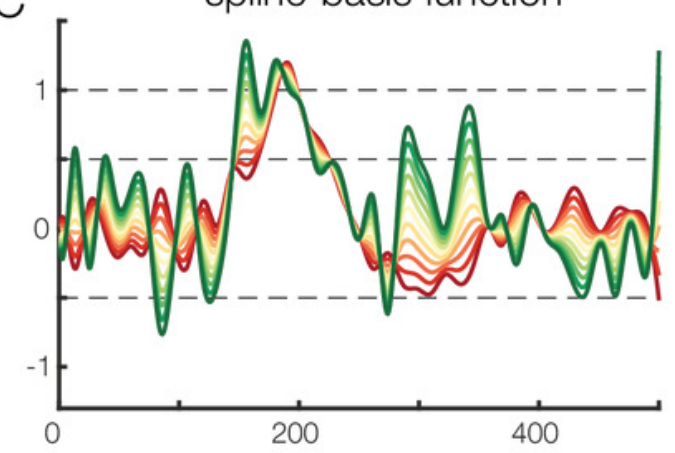

E

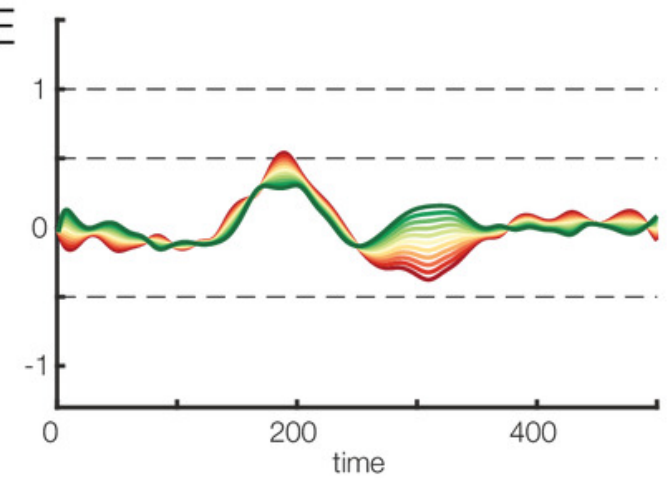




\section{Figure 7}

Regularization options.

Effects of regularization on deconvolving noisy data. Results of regularization are shown both for a model with stick-functions and for a model with a temporal spline basis set. (A) To create an overlapped EEG signal, we convolved 38 instances of the original signal depicted in panel A. The effect of a continuous covariate was randomly added to each event (see different colors in A). To make the data noisy, we added Gaussian white noise with a standard deviation of 1 . Finally, to illustrate the power of regularization, we also added another random covariate to the model. This covariate had no relation to the EEG signal but was highly correlated $(r=0.85)$ to the first covariate. Thus, the model formula was: $E E G \sim 1$ + covariate + randomCovariate. (B) Parameters recovered based on ordinary least squares regression. Due to the low signal-to-noise ratio of the data, the estimates are extremely noisy. (C) Some smoothing effect can be achieved by using time-splines as a temporal basis set instead of stick functions. (D) The same data, but deconvolved using a L2-regularized estimate (ridge regression). It is obvious that the variance of the estimate is a lot smaller. However, compared to the original signal shown in panel A, the estimated signal is also much weaker, i.e. there is a strong bias. (E) L2-regularized estimates, computed with a time-spline basis set. This panel shows the usefulness of regularization: the effect structure can be recovered despite strong noise, although the recovered effect is again strongly biased (due to the variance/bias tradeoff). 

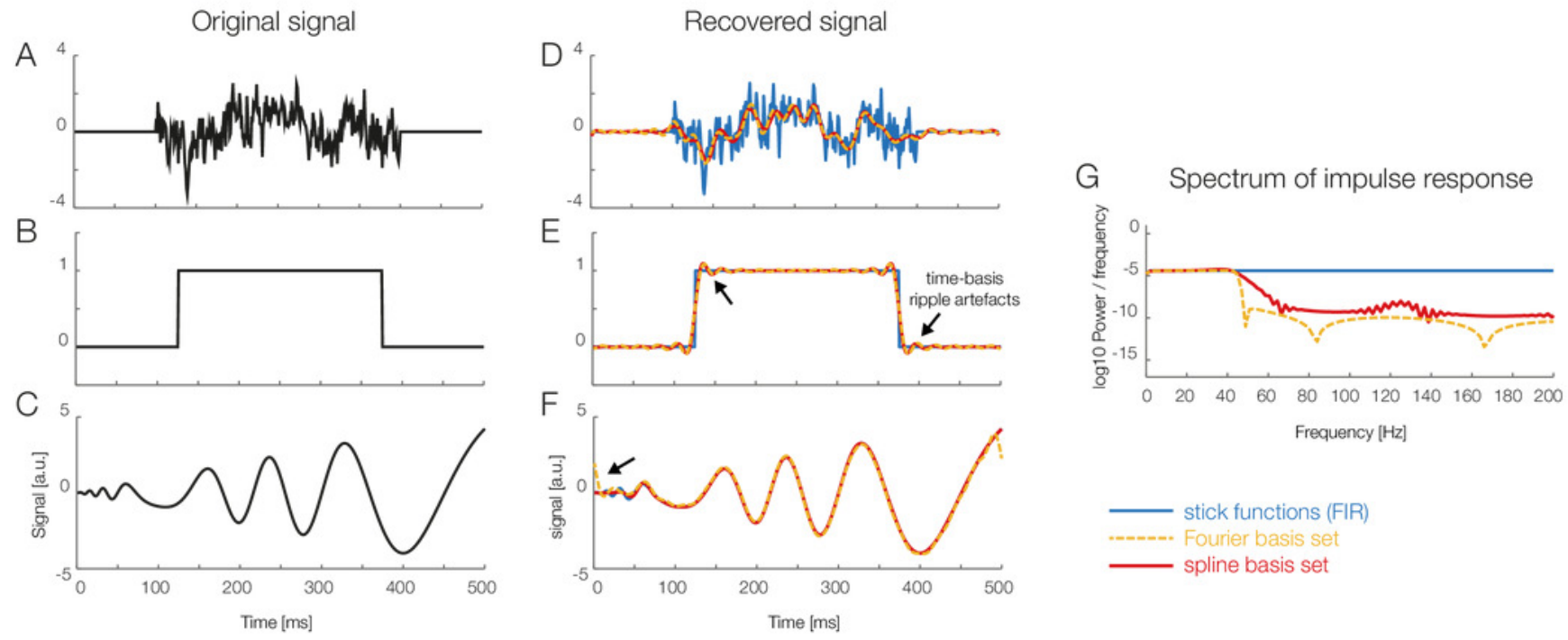

- stick functions (FIR)

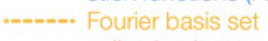

— spline basis set 


\section{Figure 8}

Inbuilt data visualization options.

Shown are some of the figures currently produced by the unfold toolbox. While setting up the model, it is possible to visualize intermediate steps of the analysis, such as the design matrix (A) covariance matrix of the predictors (B) or the time-expanded design matrix (C). After the model is computed, the beta coefficients for one or more predictors can be plotted as ERPlike waveforms with a comparison of with and without deconvolution (D), as erpimages with time against predictor value and color-coded amplitude (E), or as topographical time series (F). 

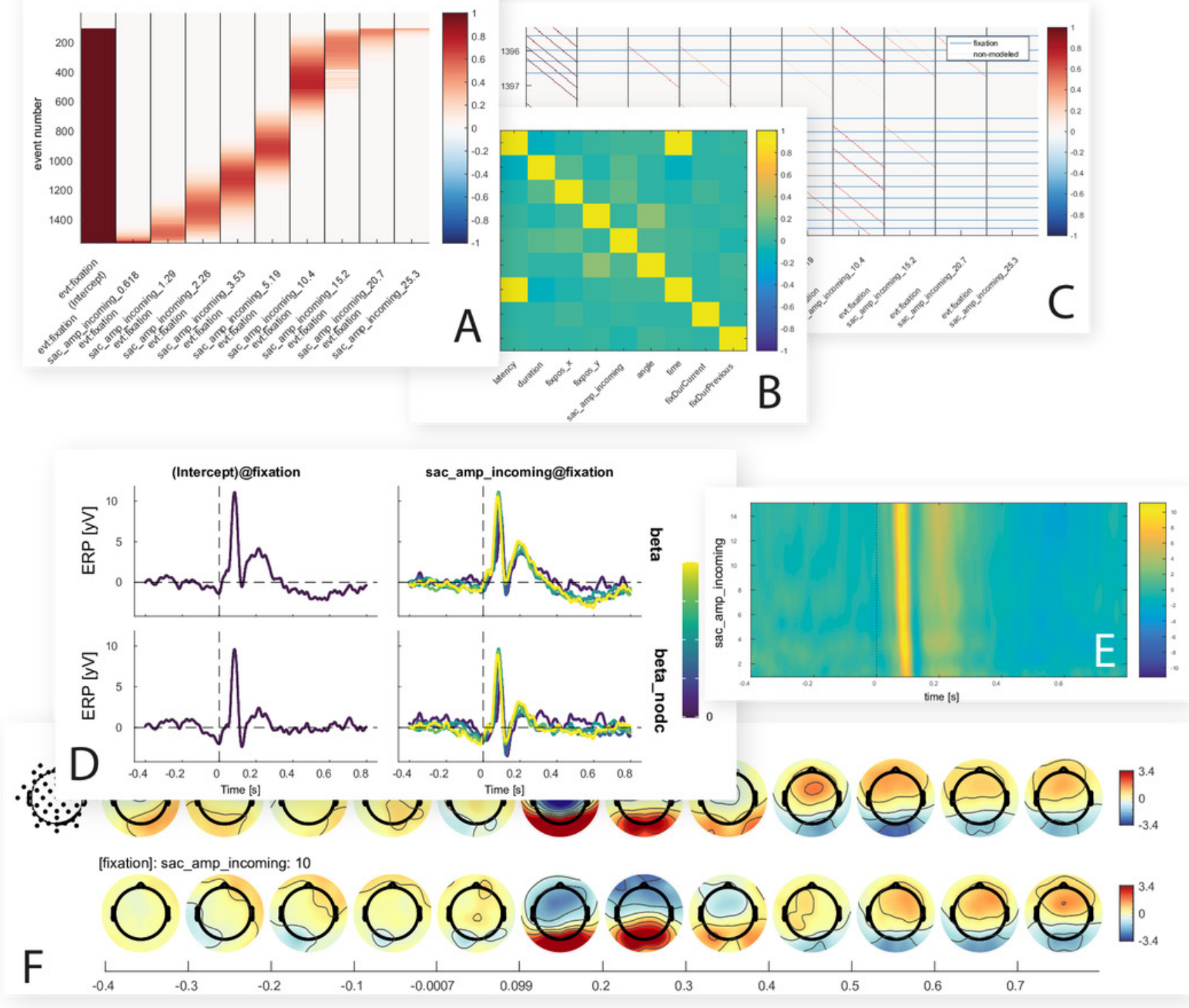


\section{Figure 9}

\section{A complete analysis script with unfold}

For further documentation and interactive tutorials visit https://www.unfoldtoolbox.org

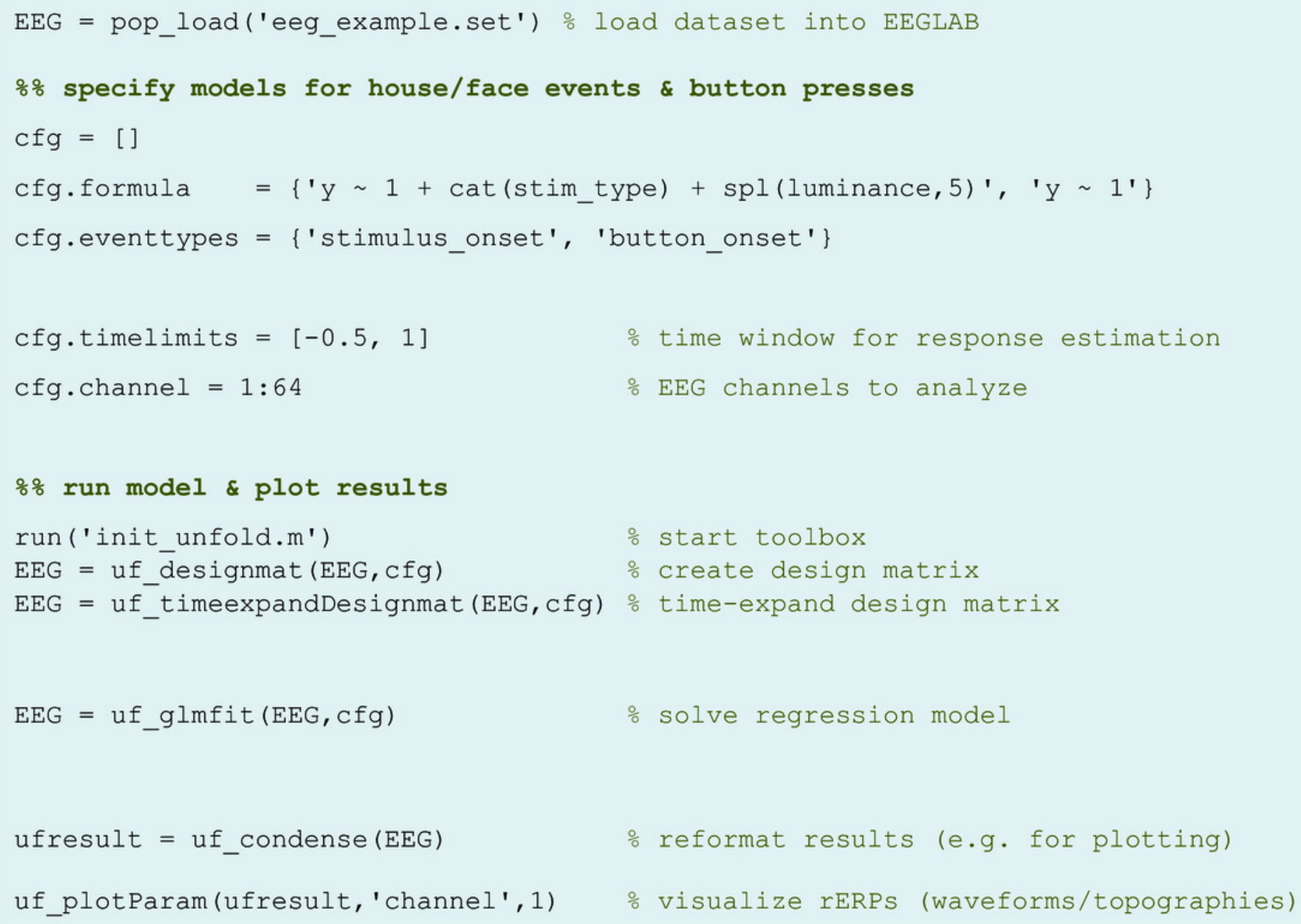




\section{Figure 10}

Deconvolution results for simulated signals.

Four types of responses (first column: box car, Dirac function, auditory ERP, pink noise) were convolved with random event latencies (second column). A section of the resulting overlapped signal is shown in the third column. The fourth column shows the deconvolved response recovered by the unfold toolbox (orange lines). Overlapped responses (without deconvolution) are plotted as violet lines for comparison.

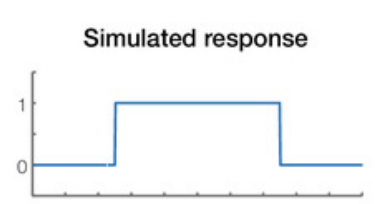

Latencies of events

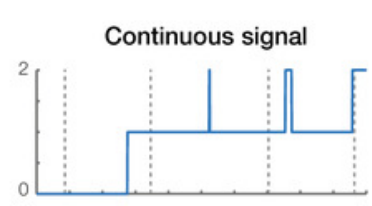

Overlapped and
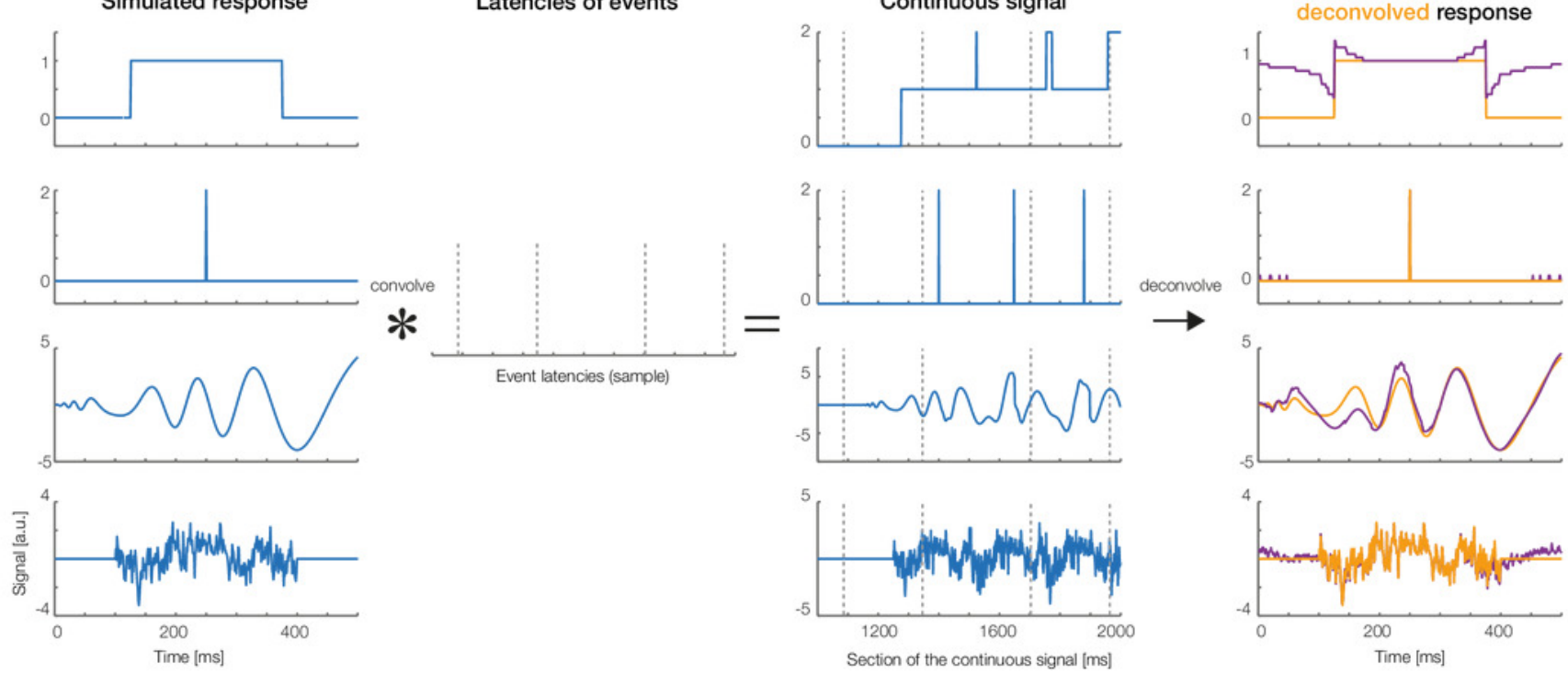


\section{Figure 11}

Example dataset with stimulus onset, eye movements and button presses

(A) Panel adapted from Dimigen \& Ehinger (2019) . The participant was shown a stimulus for 1350 ms. (B) The subject was instructed to keep fixation, but as the heatmap shown, made many small involuntary saccades towards the mouth region of the presented stimuli. Each saccade also elicits a visually-evoked response (lambda waves). (C to E) Latency-sorted and color-coded single-trial potentials at electrode Oz over visual cortex (second row) reveal that the vast majority of trials contain not only the neural response to the face $(C)$ but also hidden visual potentials evoked by involuntary microsaccades (D) as well as motor potentials from preparing the button press (E). Deconvolution modeling with unfold allows us to isolate and remove these different signal contributions (see "no deconvolution" vs. "with deconvolution"), resulting in corrected ERP waveforms for each process (blue vs. red waveforms). This reveals for example that a significant part of the P300 evoked by faces (arrow in (C)) is really due to microsaccades and button presses and not the stimulus presentation. 


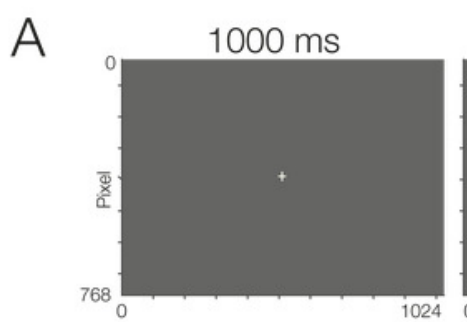

C
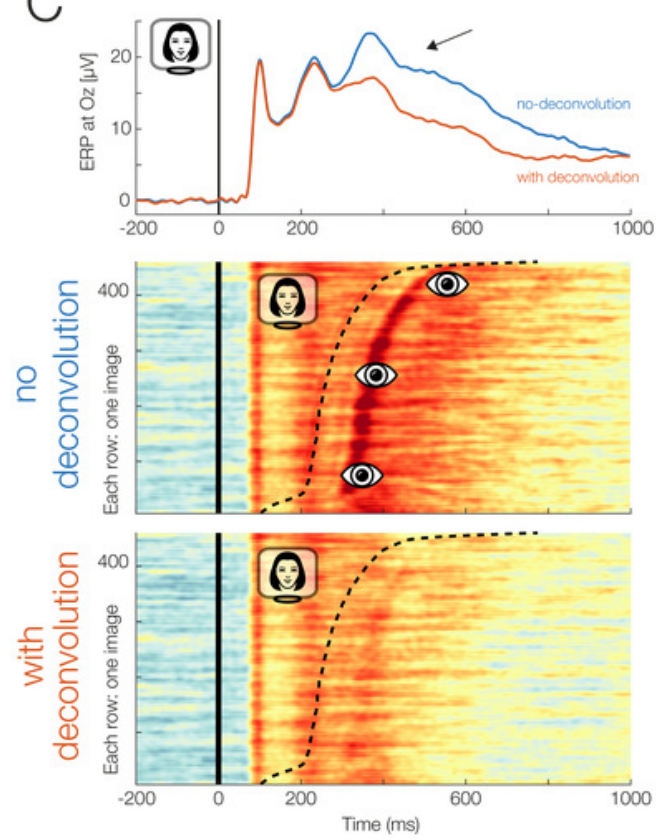

$1350 \mathrm{~ms}$

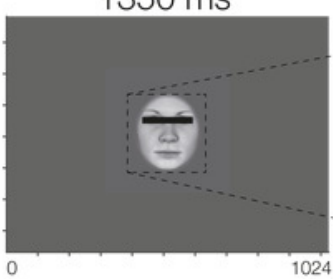

D
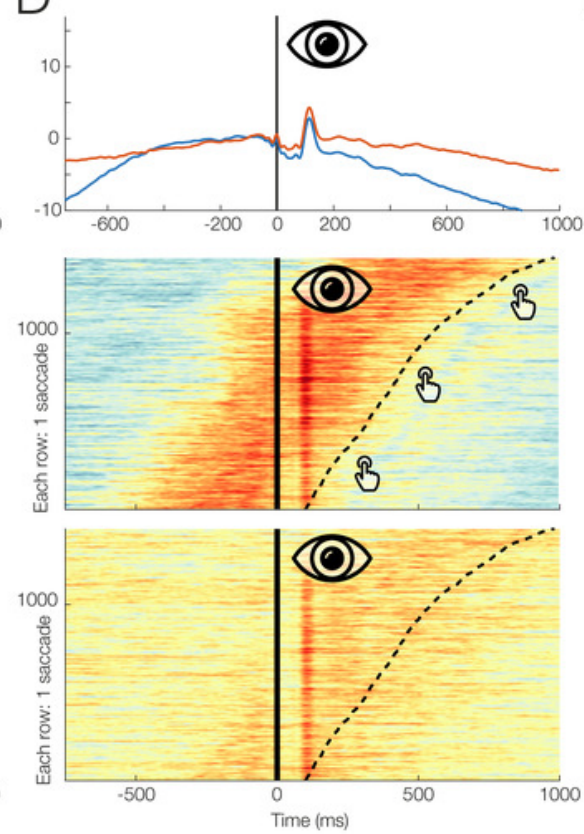

B Saccade amplitude

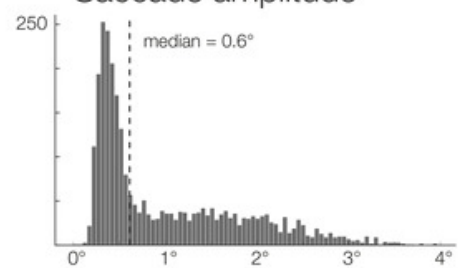

E
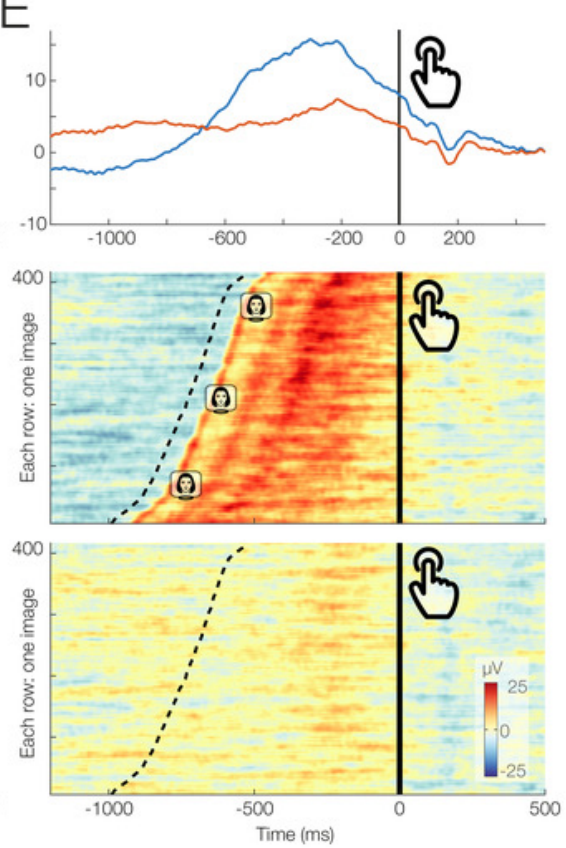